\title{
Technology Shocks and Predictable Minsky Cycles
}

\author{
Jean-Paul L'Huillier, Gregory Phelan, and Hunter Wieman*
}

January 2021

\begin{abstract}
Big technological improvements in a new, secondary sector lead to a period of excitement about the future prospects of the overall economy, generating boom-bust dynamics propagating through credit markets. Increased future capital prices relax collateral constraints today, leading to a boom before the realization of the shock. But reallocation of capital toward the secondary sector when the shock hits leads to a bust going forward. These cycles are perfectly foreseen in our model, making them markedly different from the typical narrative about unexpected financial shocks used to explain crises. In fact, these cycles echo Minsky's original narrative for financial cycles, according to which "financial trauma occur as normal functioning event in a capitalistic economy."(Minsky, 1980)
\end{abstract}

Keywords: Endogenous cycles, boom-bust dynamics, optimism, credit markets, predictability.

JEL classification: E22, E23, E32, E44

*L'Huillier: Brandeis University, 415 South Street, Waltham, MA 02453, email: jpl@brandeis.edu. Phelan: Williams College, Department of Economics, Schapiro Hall, 24 Hopkins Hall Drive, Williamstown MA 01267, email: gp4@williams.edu. Wieman: Williams College, email: hlw2@williams.edu. We are grateful for feedback from Matthew Rognlie, Alp Simsek, Mathieu Taschereau-Dumouchel, Kieran Walsh, Xian Wang, and seminar participants at the Tinbergen Institute. All errors are our own. 


\section{Introduction}

There is an old idea in macroeconomics that major technological advances generate dramatic boom-bust cycles that typically finish in a pronounced and somewhat predictable financial retrenchment. There is growing recent evidence that market economies are subject to endogenous boom-bust cycles, with times of expansion "sowing the seeds" for the slump that follows (see Beaudry et al., 2017, 2020). In fact, the Great Recession, the Great Depression, and the Japanese slump of the 1990s were all preceded by periods of major technological innovation (Cao and L'Huillier, 2018), and it is easy to find similar evidence for the case of sudden-stops in emerging markets (Boz, 2009). But these episodes were not just preceded by positive technological innovation. The observed realizations of permanent technology shocks during these episodes exhibit a strong and persistent increase of productivity growth rates and an equally strong reversal. ${ }^{1}$ Positive productivity shocks are followed by equally negative productivity "shocks." While the good-news-bad-news pattern could just be random, such consistent evidence across episodes suggests that positive productivity shocks sow the seeds for trouble.

In addition to seeing evidence for boom-bust dynamics in productivity shocks, it is well known that these major episodes often feature credit expansions during the boom and contractions, or even financial crises, during the slump. Economists since Fisher (1933), Keynes (1936), and Minsky $(1982,1986)$ have seen the behavior of financial markets as playing a central role in economic downturns. There remains considerable debate about the causes and consequences of recessions, and still less is known about the role, if any, of endogenous boom-bust dynamics.

In light of the empirical evidence and stylized facts regarding the role of positive productivity, credit expansions, and reversals in both productivity and credit, we consider a model that can explain the dynamics that appear in the data surrounding major boom-bust episodes. We consider a standard model with collateral constraints following Kiyotaki and Moore (1997) and consider news of a reallocative technology shock: a positive technology shock that does not primarily affect the most productive users of capital (who invest with leverage) and so leads to a future reallocation of resources away from these leveraged users. Our model features perfect foresight and rational expectations and is thus not driven by sluggishness in beliefs or failure to anticipate the equilibrium consequences of the productivity shock. Thus, the mechanisms of the "Minsky Cycle"

${ }^{1}$ Cao and L'Huillier (2018) estimate positive shocks from roughly 1989 to 1999, and negative shocks later on. They find fairly strong evidence in the data of either a large positive shock or several positive shocks somewhere in the early 9os, although it is not possible to know exactly when. The opposite holds starting in 1999 . 
in our model are quite distinct from the "Wile E. Coyote" moment in the literature (see Eggertsson and Krugman, 2012), and financial retrenchment in our model is completely predictable.

Our paper offers a parsimonious and internally consistent model to rationalize macrofinancial boom-bust cycles. The narrative we have in mind is as follows. The economy experiences news of a positive productivity boom in some new technology or secondary sector, which will lead to a reallocation of resources toward the new technology. In anticipation of future growth, asset prices increase right away, which fuels a credit expansion affecting the entire economy, not just the sector that will experience the technological innovation. This credit-filled boom is primarily driven by leveraged users of capital. However, the positive shock in the new sector pulls resources away from the economy's primary producers, who have taken on more debt during the credit expansion, and the primary producers are forced to cut their capacity to repay debts. This deleveraging process leads to a persistent bust after the transitory positive shock dissipates. Our narrative matches the stylized facts of emerging market "sudden stop" episodes, as well as for the Great Recession in the U.S., which was preceded by new innovations in the Information Technology sector as well as a boom in the housing market. ${ }^{2}$

There are several reasons why news of a reallocative technology shock is an attractive candidate to make sense of Minsky Cycles. First, news of a future reallocation endogenously leads to boom-bust dynamics in asset prices and output. Additionally, a reallocative technology shock can lead output to fall while asset prices are still high. This disconnect between real and financial variables together with the subsequent convergence generates predictable dynamics similar to a "Minsky Moment" when asset prices suddenly "correct" after an unsustainable period of exuberance. Second, a reallocative techonology better matches the dynamics of a Minsky cycle than a technology shock that primarily affects the leveraged users. A technology shock that primarily affects the leveraged users of capital leads unequivocally to a persistent boom. In order for a bust to occur, good news must be followed by bad news, in which case the shocks are truly driving the "cycle" rather than endogenous dynamics. Finally, and perhaps most importantly, a reallocative technology shock is a much better candidate than a shock to financial conditions directly. A reallocative technology shock produces dynamics reminiscient of Minsky's narrative, but a shock relaxing financial conditions directly (e.g., a

${ }^{2}$ For simplicity, we start with a transitory shock as a way of capturing the dynamics that typically occur when a new technology or innovation arises and investment flows in quickly, perhaps exceeding the steady-state level. However, whether the shock is transitory or permanent does not matter for the argument, as we show in an extension. Our focus in not on modeling the behavior of investment in the innovative technology, but to focus on how such an innovation affects the broader economy. 
"financial liberalization") produces very different dynamics in output and asset prices. A temporary relaxation of collateral constraints produces an endogenous cycle without an increase in asset prices (asset prices stay the same and then fall going forward), but high asset prices are central to the narratives of Minsky, Fisher, and Keynes.

\section{Related Literature}

We are far from the first paper to consider endogenous boom-bust dynamics in market economies. Beaudry et al. $(2017,2020)$ provide evidence of medium-run cyclical behavior in aggregate variables. Beaudry et al. (2018) propose a model that includes Hayekian mechanisms of over-investment and liquidation with Keynesian mechanisms working through aggregate demand. Rognlie et al. (2018) consider how over-investment in one sector (as an initial condition) together with nominal rigidities at the ZLB lead to investment hangover during the recovery. We show how initial over-investment is likely to occur given the nature of productivity news we think is relevant in the data.

Our analysis is most closely related to Kiyotaki and Moore (1997), who extend the insight from Bernanke and Gertler (1989) that changes in borrower net wealth and agency costs create persistence in business cycles, to show that borrowing constraints also amplify business cycles precisely because the values of borrowers' assets are pro-cyclical. Kiyotaki and Moore (1997) consider a temporary shock to productivity that affects all agents, but most importantly the shock increases the funds available to experts ("farmers," in their terminology). The initial increase in output leads experts to buy more capital, increasing their output next period, and increasing asset prices next period. The increase in future asset prices relaxes current collateral constraints, leading to amplifications in current output and asset prices that are an order of magnitude larger than would occur in a frictionless model. An extended version of the model with investment features internal propagation mechanisms that can lead to credit cycles in response to the aforementioned shock. However, when such models are estimated, the implied parameters generally do not generate quantitatively meaningful endogenous cyclical behavior. In contrast, our model generates cyclical behavior in the baseline Kiyotaki and Moore (1997) setup because of the timing and nature of the productivity shock. The bust following the shock is of the same order of magnitude as the initial shock itself and is not driven by amplification mechanisms, but by reallocations that occur to the new sector and following expansion by the main sector during the credit expansion.

Closely related to our focus on collateral constraints, Akınc1 and Chahrour (2018) consider an open economy with occasionally binding collateral constraints and find that 
positive productivity shocks increase leverage, thus increasing the probability of a future Sudden Stop. On average good news is realized, but higher leverage exposes agents to a greater risk that an unfavorable future shock will eventually lead the constraint to bind. Our model considers a single positive productivity shock and does not rely on the possibility of unfavorable future shocks. Bhattacharya et al. (2015) provide a model of rational learning in which periods of good times leads to more optimism and greater leverage. Eggertsson and Krugman (2012) assume a "Minsky moment" when borrowing constraints suddenly tighten and study the aggregate consequences. Farhi and Werning (2020) study optimal coordination of monetary and macroprudential policy when Minsky cycles are caused by excessive optimism (extrapolative expectations). Gorton and Ordonez (2020) find support that financial cycles can be thought of as medium-run phenomena.

Empirical evidence supports the pattern of boom-bust investment cycles as well as the predictability of asset price busts (or financial crises). Gulen et al. (2019) find that elevated credit-market sentiment correlates with a boom in corporate investment over the subsequent year, followed by a long-run contraction. López-Salido et al. (2017) find that elevated credit-market sentiment predicts lower GDP growth two years later. Greenwood et al. (2019) find that financial fragility arises at the end of economic expansions and Greenwood et al. (2020)find that financial crises are predictable, as in our paper.

\section{The Model}

The baseline model is identical to the model proposed by Kiyotaki and Moore (1997). In section 2.2 we introduce the shock consistent with our narrative: positive news about a technology shock to an innovative, secondary sector.

\subsection{Baseline Model}

Setup Time is discrete and infinite. The economy contains a single durable factor of production, which we call capital. The aggregate supply of capital is fixed at $\bar{K}$. Capital trades at a price $q_{t}$ per unit of output.

There are two types of agents, experts and non-experts, who for simplicity have linear utility over consumption. Non-experts discount future consumption using discount factor $\underline{\beta}$ (we underline non-expert variables). Experts are strictly more impatient. 
Technology There are two types of production technologies. Non-experts have production function $G$ with decreasing returns to scale: a non-expert with $\underline{k}_{t}$ units of capital at $t$ produces

$$
\underline{y}_{t+1}=G\left(\underline{k}_{t}\right)
$$

units of output in $t+1$. Experts have linear technology: an expert with $k_{t}$ units of capital at $t$ produces

$$
y_{t+1}=(a+c) k_{t}
$$

units of output in $t+1$, where $a k_{t}$ units are tradable and can be used to purchase capital but $c k_{t}$ units are non-tradable and must be consumed by experts. As in Kiyotaki and Moore (1997), we suppose that $c$ is sufficiently large relative to the experts' discount factor so that experts will not consume any of the tradable output. As explained below, experts are subject to a collateral constraint limiting their credit, which will bind in equilibrium. We make assumptions on $G$ so that, in equilibrium, experts' marginal productivity is above non-experts' and thus the optimal allocation gives capital to experts.

Budget and Collateral Constraints All borrowing must be collateralized by capital. Since experts are more productive and more impatient, experts will borrow from nonexperts in equilibrium. At date $t$ an expert with capital $k_{t}$ can borrow up to the value of the capital in $t+1$, i.e.

$$
R b_{t} \leq q_{t+1} k_{t}
$$

where $R$ is the gross interest rate, $b_{t}$ is the amount borrowed, $q_{t+1}$ is the future asset price, and $k_{t}$ are present capital holdings. Because non-experts are unconstrained, their discount factor pins down the rate to $R=1 / \underline{\beta}$. An expert borrowing $b_{t}$ at interest rate $R$ must repay $R b_{t}$ tomorrow. The capital tomorrow has value $q_{t+1} k_{t}$.

Given the assumptions, experts borrow up to the collateral constraint and use all tradable output to buy capital. An expert's budget constraint is

$$
q_{t} k_{t}=\left(a k_{t-1}+q_{t} k_{t-1}-R b_{t-1}\right)+b_{t} .
$$

Plugging in for $b_{t}$ using the collateral constraint yields

$$
k_{t}=\frac{\left(a k_{t-1}+q_{t} k_{t-1}-R b_{t-1}\right)}{q_{t}-\frac{q_{t+1}}{R}}=\frac{\left(a k_{t-1}+q_{t} k_{t-1}-R b_{t-1}\right)}{u_{t}},
$$

where $u_{t} \equiv q_{t}-\frac{q_{t+1}}{R}$ is the user cost or the down payment for a unit of capital. 
Non-experts are not credit constrained, which means they will hold capital until the marginal value of capital equals the opportunity cost $R$ :

$$
R=\frac{G^{\prime}\left(\underline{k}_{t}\right)+q_{t+1}}{q_{t}}, \Longrightarrow \frac{1}{R} G^{\prime}\left(\underline{k}_{t}\right)=u_{t} .
$$

Aggregate Equations By linearity, we can aggregate by summing over experts to get

$$
\begin{aligned}
K_{t} & =\frac{1}{u_{t}}\left(a K_{t-1}+q_{t} K_{t-1}-R B_{t-1}\right), \\
B_{t} & =\frac{q_{t+1} K_{t}}{R},
\end{aligned}
$$

where $K_{t}$ and $B_{t}$ are aggregate capital holdings and borrowing by experts. The user cost is given by

$$
u_{t}=\frac{1}{R} G^{\prime}\left(\bar{K}-K_{t}\right) .
$$

Total output is given by

$$
Y_{t+1}=(a+c) K_{t}+G\left(\bar{K}-K_{t}\right) .
$$

In the steady-state equilibrium we have

$$
u^{*}=a, \quad q^{*}=\frac{a R}{r}, \quad R a=G^{\prime}\left(\bar{K}-K^{*}\right), \quad Y^{*}=(a+c) K^{*}+G\left(\bar{K}-K^{*}\right),
$$

where $r=R-1$ is the net interest rate. Thus, the tradable output just covers the interest on experts' debt, and the down payment equals the tradable output.

\subsection{News Shocks to an Innovative Sector}

Central to our boom-bust narrative is that the positive productivity shock leading to the expansion affects some innovative or secondary sector. In light of the behavior of the motivating events as discussed in the Introduction, we view this innovation as offering temporarily higher productivity, Initially, this innovative sector is secondary to the most productive uses of capital in the economy, though in reality the most productive uses of capital may also benefit from this sector (examples include IT, innovations in housing finance, or real estate more broadly).

Our goal is to present a simple model that can clarify the interaction of optimism with capital reallocation, and how it has the ability to generate predictable financial cycles. Since in reality there are many factors driving optimism, credit expansion, and 
reallocation, we remain agnostic about how exactly to map the innovative sector to any given episode. We prefer to interpret our model as highlighting the key mechanisms at play in these events. Accordingly, we model the productivity shock of interest as the temporary entrance of an innovative technology. ${ }^{3}$ The innovative technology is a linear production function

$$
y_{t+1}=a^{I} k_{t}^{I}
$$

with $a+c>a^{I}>R a$, and supserscript $I$ denotes variables associated with the innovative technology. The productivity of the innovative technology is higher than non-experts' marginal productivity in steady state but lower than the experts'. ${ }^{4}$

We suppose that in a future period $t^{\prime}$, non-experts have access to this more-productive innovative technology for one period. For simplicity, we suppose each non-expert has access to the innovative technology but with a capacity constraint. Since the innovative technology has a higher marginal product that $G$ in steady state, non-experts will invest as much as possible in the new technology. Hence, we directly specify the shock as the quantity of capital $K^{I}$ that gets invested using the innovative technology. Aggregate output at $t^{\prime}$ is therefore given by

$$
Y_{t^{\prime}+1}=(a+c) K_{t^{\prime}}+a^{I} K^{I}+G\left(\bar{K}-K_{t^{\prime}}-K^{I}\right) .
$$

Crucially, a credit expansion requires that future asset prices increase. For this reason, at time $t$ agents receive news of the future innovative technology at $t^{\prime}$. Since in our main specifications the technology is only available for one period, we refer to access to the innovative technology in period $t^{\prime}$ as as "the shock."

\subsection{Linearized Equilibrium}

Similar to Kiyotaki and Moore (1997), we solve for the log-linearized dynamics around the steady state. For a variable $X_{t}$, we denote log-deviations from steady state by $\hat{X}_{t} \equiv \log \left(X_{t}\right)-\log \left(X^{*}\right)$ where $X^{*}$ denotes the steady-state value. (We use the terms "log-linearization" and "linearization" interchangeably.) Following the same notation as Kiyotaki and Moore (1997), let $1 / \eta$ denote the elasticity of the user cost to changes in

\footnotetext{
${ }^{3}$ This is only for simplicity, in an extension we show that this can generalize to permanent shocks, as well.

4Since the innovative technology is run by non-experts, who are unconstrained, we need not distinguish between tradable and non-tradable production for the innovative technology. However, it is sensible to suppose that $a k_{t}^{I}$ units are tradable so that the innovative technology has the same marginal product of tradable output as the expert technology.
} 
aggregate non-expert capital. By definition, $\eta=-\frac{G^{\prime}\left(\bar{K}-K^{*}\right)}{G^{\prime \prime}\left(\bar{K}-K^{*}\right) K^{*}}$, where $K^{*}$ is capital held by experts. ${ }^{5}$ It is a convenient normalization to directly define the demand shock for innovative capital $\hat{K}^{I}$ as a fraction of experts' steady-state capital holdings, i.e., total capital demand $K^{I}=K^{*} \hat{K}^{I}$. Accordingly, we also define $\hat{z}=\hat{K}^{I} / \eta$, which is the change in non-experts' marginal product (the change in $G^{\prime}$ ) when shifting $K^{I}$ units of capital away from their primary production technology and towards the innovative technology.

Since the behavior of asset prices is crucial for our story, we provide the linearized equations for the asset price here to emphasize how the shock affects prices each period. The remaining equations are in the appendix. Linearizing the non-experts' optimality condition in equation (4) delivers

$$
\hat{u}_{t}=\frac{1}{\eta} \hat{K}_{t}, \forall t \neq t^{\prime}, \quad \text { and } \quad \hat{u}_{t^{\prime}}=\frac{1}{\eta} \hat{K}_{t^{\prime}}+\hat{z},
$$

where $\hat{K}_{t}$ is aggregate expert capital holdings and $\hat{z}=\hat{K}^{I} / \eta$ is the shock at $t^{\prime}$. The only mechanism affecting the user cost is the change in non-expert capital. In the absence of the shock, non-experts hold less capital when experts hold more, and the user cost increases since non-experts' marginal productivity rises. However, in the period of the shock $t^{\prime}$, capital is allocated to the innovative technology and so non-experts have higher marginal productivity when using $G$ with less capital.

From the definition of the user cost, we can write $q_{t}=u_{t}+\frac{q_{t+1}}{R}$. Linearizing we have

$$
\hat{q}_{t}=\frac{r}{R} \hat{u}_{t}+\frac{1}{R} \hat{q}_{t+1}=\frac{r}{R} \sum_{s=0}^{\infty} \underline{\beta}^{s} \hat{u}_{t+s}
$$

where the last line follows from forward iteration.

\section{Results}

For expositional clarity, our main results consider a one-time impulse shock at a time $t^{\prime}$. In this section we let the economy start in equilibrium at $t=0$ and suppose that the innovative technology is available in one period $\left(t^{\prime}=1\right)$. Section 4 considers the case when the shock occurs $N>1$ periods forward $\left(t^{\prime}=N\right)$ implying a greater role for news and anticipation, and also considers persistent shocks. Section 5 discusses the robustness of our results by considering the role of news, expectations, general equilibrium

\footnotetext{
${ }^{5}$ With Cobb-Douglas productivity $G(\underline{k})=z \underline{k}^{\alpha}$, at steady-state we have $\eta=\left(\frac{1}{1-\alpha}\right)\left(\frac{\bar{K}-K^{*}}{K^{*}}\right)$.
} 
adjustments, and alternative sources of shocks.

\subsection{Dynamics for Shock in One Period, $t^{\prime}=1$}

After news of the shock has been incorporated, experts' borrowing in future periods equals the value of capital in the next period. In contrast, at $t=0$ news of the shock can increase the value of capital at $t=0$ so that it exceeds the debt that needs to be repaid (experts' borrowing is inherited from the previous period, which was determined before news of the shock). We can unequivocally describe the deterministic behavior of capital and asset prices arising due to the change in productivity happening at $t=1$ as a result of the innovative sector. Define $\gamma \equiv \frac{1}{\left(1+\frac{1}{\eta}\right)}=\frac{\eta}{1+\eta}$, which reflects the price elasticity of non-expert demand for capital; $\gamma<1$ since $\eta>0$.

Proposition 1. In response to a news shock at $t$ regarding the productivity of the innovative sector at $t+1$, the economy experiences the following deterministic boom-bust dynamics:

1. An increase in the capital price at $t=1: \hat{q}_{1}=r \gamma \beta \hat{z}>0$,

2. A boom at time $t=0: \hat{K}_{0}=\underline{\beta} \gamma \hat{z}>0$ and $\hat{q}_{0}=r \underline{\beta}^{2} \hat{z}>0$,

3. A bust going forward: $\hat{K}_{s}=\gamma^{s}\left(\hat{K}_{0}-\hat{z}\right)<0$ for all $s \geq 1$, with $\hat{q}_{s}<0$ for all $s \geq 2$.

The demand for capital from the innovative sector will increase the asset price at $t=1$, which relaxes collateral constraints at $t=0$ and increases experts' capital holdings right away. Experts' demand for capital at $t=0$ increases the asset price and the user cost above the steady state value. But this means that experts' debt exceeds the sustainable steady state level (in which $u_{t}=a$ ). In contrast to Kiyotaki and Moore (1997), experts are not more productive at $t=1$ as a result of the shock. Experts have higher output because they held more capital, but their debt burdens are even higher, and since the user cost exceeds the value of their output, experts must sell capital at $t=1$ in order to repay their debts.

Since experts' debt is higher but their productivity is not, experts are forced to sell capital to the innovative sector but also to non-experts, pushing their capital holdings below the steady-state level. Once experts' capital is below the steady state, experts slowly rebuild capital as they pay off their debts. Accordingly, the economy will experience a boom-bust cycle arising from the initial relaxation of constraints and the subsequent tightening that forces experts to sell capital to non-experts. ${ }^{6}$

\footnotetext{
${ }^{6}$ Different from Kiyotaki and Moore (1997), we do not see amplification in response to a shock to
} 
Output and Aggregate Productivity Since the shock is an exogenous demand for capital, the dynamics of capital allocations and prices are independent of the innovative productivity $a^{I}$. However, output at the time of the shock depends critically on $a^{I}$.

In this simple model aggregate capital is fixed, and thus fluctuations in output reflect changes in productivity (i.e., changes in capital allocation). When $t \neq t^{\prime}$, any change in output next period is driven by changes in expert (non-expert) capital holdings:

$$
\hat{Y}_{t+1}=(a+c-R a) \frac{K^{*}}{Y^{*}} \hat{K}_{t}
$$

The percent change in output reflects the productivity difference between experts and households $a+c-R a$, times the share of output their capital creates $\frac{K^{*}}{Y^{*}}$ times the change in capital $\hat{K}_{t}$.

At $t^{\prime}+1$, output is also affected by the capital holdings of the innovative sector at $t^{\prime}$ :

$$
\hat{Y}_{t^{\prime}+1}=(a+c-R a) \frac{K^{*}}{Y^{*}} \hat{K}_{t^{\prime}}+\left(a^{I}-R a\right) \frac{K^{*}}{Y^{*}} \hat{K}^{I} .
$$

Output changes for two reasons: experts have additional capital $\hat{K}_{t^{\prime}}$, which increases productivity relative to households by $a+c-R a$, and the innovative sector has additional capital $\hat{K}^{I}$, which increases productivity relative to households by $a^{I}-R a$, and both terms are weighted by the capital share.

The following result is an immediate implication of equation (14) and the fact that $\hat{K}_{t}$ is independent of $a^{I}$.

Proposition 2. In response to a news shock at $t=0$ regarding the productivity of the innovative sector at $t=1$, such that the economy experiences the following deterministic boom-bust dynamics: A boom at time $t=1: \hat{Y}_{1}>0$; a bust going after the shock: $\hat{Y}_{s+1}<0$ for all $s \geq 2$; furthermore, there exists a maximum productivity $\bar{a}^{I}$ such that the economy experiences a bust at $t=2$ if and only if $a^{I}<\bar{a}^{I}$.

Figure 1 illustrates the results. The figure plots the equilibrium dynamics for experts' capital holdings, capital prices, and output next period in response to a shock $\hat{z}=1 \%$ at $t=1.7$ Capital initially increases and then falls at the time of the shock (in period 2), slowly returning to steady state. The capital price $q_{t}$ is above steady state for 2 non-experts' productivity. The increase in capital is of the same order of magnitude at $\hat{z}$, while the increase in the capital price is an order of magnitude smaller. Thus, the model creates boom-bust, but not amplification.

7We parameterize with $\alpha=0.3, a=z=0.3, c=0.3, \bar{a}=0.4$, and $R=1.02$. We calibrate the steady-state expert share of capital to be $25 \%$ of total capital. 
periods before falling below steady state, while output next period falls below steady state even in the time of the shock (since output is completely determined by variables in the previous period, we choose to plot output next period as a function of time). As a result, we have a divergence in output and capital prices when the shock hits: capital prices remain above steady state even though output falls below.

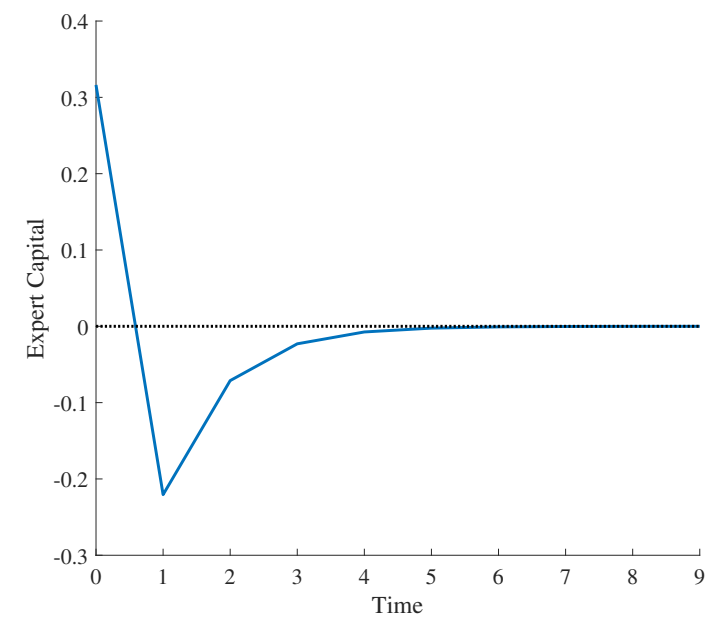

(a) Experts' Capital

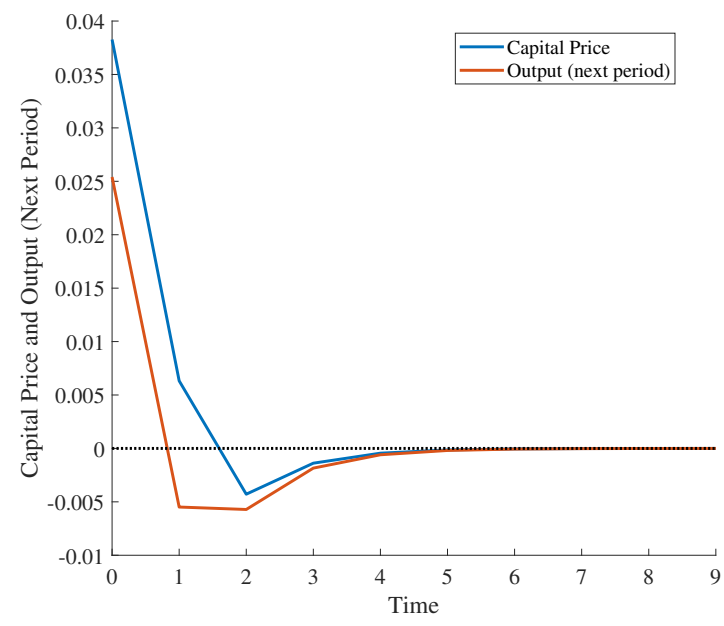

(b) Capital Prices and Output

Figure 1: Changes in experts' capital $\hat{K}_{t}$, capital price $\hat{q}_{t}$, and output $\hat{Y}_{t+1}$ in response to news at $t=0$ of an innovative sector at $t=1$ (because of the timing convention in the model, capital at $\mathrm{t}=\mathrm{O}$ is used to produce $\mathrm{t}=\mathrm{I}$ output)

A crucial parameter for the boom-bust dynamics of output/productivity is the productivity of innovative technology, $a^{I}$, which determines the severity of the boom or bust at $t^{\prime}$ only. If $a^{I}$ is not so large compared to the productivity of the experts (if $a^{I}$ is sufficiently less than $a+c$ ), then the shock leads to output below steady state at $t^{\prime}$ : experts hold less capital than steady state, and even though the innovative technology is marginally more productive than $G$, aggregate productivity falls because the innovative technology is so much less productive than experts'. However, if $a^{I}$ is sufficiently close to $a+c$ (not necessarily more), aggregate productivity can be above steady state at the time of the shock. The economy will still feature a boom-bust cycle in output, with productivity falling below steady state in the periods after the shock, but the boom will decline more slowly. 


\section{Prolonged Anticipation and Persistent Shocks}

We now suppose that the innovative technology is available in $N>1$ periods $\left(t^{\prime}=N\right)$, leading to prolonged anticipation of the reallocative technology shock. News about an event further in the future will have distinct consequences for the size of the bust when the reallocation of capital occurs at $t=N$. Finally, we consider a slowly-decaying AR-1 shock. The results extend the insights from the previous two analyses using one-time impulse shocks in the future. Appendix B.3 considers a permanent shock.

\subsection{Dynamics for $N$-period Forward Shocks, $t^{\prime}=N$}

We now consider the dynamics when agents receive news at time $t=0$ that an innovative technology will be available at time $t=N$. In contrast to the previous analysis, the initial expansion will slowly decay (at a rate determined by the elasticity $\eta$ ) as experts repay their debts from the initial expansion. However, the reallocation at $t=N$ will have a greater effect on the slump going forward since the boom will have dissipated.

Proposition 3. In response to a news shock at $t=0$ regarding the innovative technology at $t=N$, the economy experiences the following boom-bust dynamics:

1. An increase in the capital price at $t=N: \hat{q}_{N}=r \underline{\beta} \hat{z}\left(\frac{(\underline{\beta} \gamma)^{N}+\eta-\underline{\beta} \eta}{1+\eta-\underline{\beta} \eta}\right)>0$,

2. A boom before $t=N: \hat{K}_{0}=\hat{z} \underline{\beta}^{N} \gamma>0$ and $\hat{K}_{s}>0$ for $s<N$, decaying at rate $\gamma$, and $\hat{q}_{0}=r \underline{\beta}^{N+1} \hat{z}>0$, with $\hat{q}_{s}>0$ for $s<N+1$,

3. A bust going forward: $\hat{K}_{N}=-\hat{z} \gamma\left(1-(\underline{\beta} \gamma)^{N}\right)<0$, decaying at rate $\gamma$, with $\hat{q}_{N+s}<$ 0 for all $s \geq 1$.

Both $\hat{K}_{0}$ and $\hat{K}_{N}$ are decreasing in $N$, which implies a much larger slump when the innovative sector enters. The initial boom is smaller because, due to the interest rate, the effects of future increases in prices on relaxing collateral constraints gets discounted. However, the reallocation $\hat{K}_{N}$ at $t=N$ becomes more negative because the initial boom decays.

Figure 2 plots experts' capital holdings and output in response to such a shock $N$ periods forward with $N=1, \ldots, 5$. Note that the initial boom gradually decays, with greater decay the longer forward is the true shock. Accordingly, the bust is more severe, and the slump more prolonged, when the news is about events further in the future. (Since $\underline{\beta}$ is close to 1 , the initial boom is essentially the same across all cases.) 


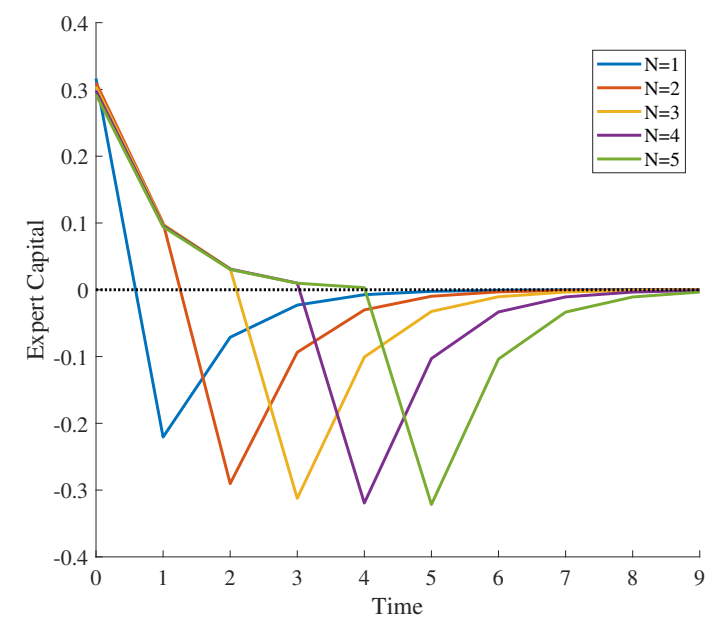

(a) Experts' Capital $\hat{K}_{t}$

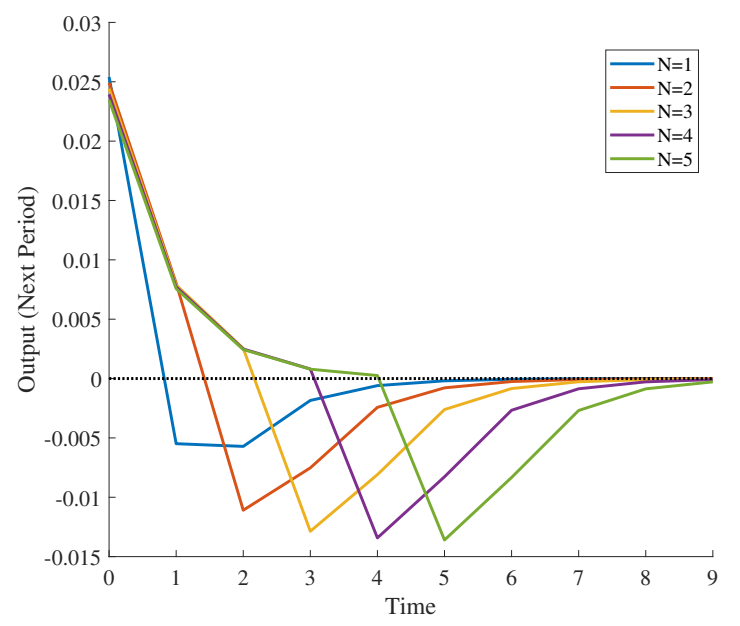

(b) Output next period $\hat{Y}_{t+1}$

Figure 2: Changes in experts' capital and output next period in response to news of an innovative sector at $t=N$, varying $N$.

The behavior of the economy in response to news about the future best illustrates Minsky's hypothesis. The boom declines in response to credit tightening: asset prices gradually decline, tightening collateral constraints, and experts are forced to decrease their capital holdings in response to tighter credit. The longer that credit tightening persists, the less experts are able to hold on to capital when the innovative sector demands it. As a result, there is a larger reallocation and a deeper, more persistent bust.

Because our model features perfect foresight in response to a one-time shock, the counterfactual result from the model is that the boom is immediate and greatest at the time of news. In reality, the economy appears to take time to learn about the news and thus slowly adjust upward to the values plotted in Figure 2. A learning model as in Blanchard et al. (2013) or Cao and L'Huillier (2018) would improve the dynamics of the model in this regard.

\subsection{Persistent or Permanent Shocks}

In this section we consider a slowly-decaying shock occurring at $t=1$. In reality shocks are likely to have a persistent component. Since the model is linearized, the dynamics in response to a persistent shock are merely the sum of the dynamics in response to the individual shocks, and therefore the response to a decaying shock combines the earlier analyses. Considering a slowly decaying shock strengthens our results, leading to more persistent busts following the boom. Appendix B considers shocks occurring further in 
the future as well as permanent shocks.

Let's suppose that starting at $t=1$ the economy experiences an AR-1 decaying shock $\hat{z}_{s}=\rho^{s-1}$, with $\rho \in(0,1)$. We have $\hat{z}_{1}=1$ and then the shock decays at rate $\rho$ going forward. Then the initial capital boom is given by

$$
\hat{K}_{0}=\underline{\beta} \gamma\left(\frac{1}{1-\underline{\beta} \rho}\right)>0 .
$$

Note that the shock to capital at $t=0$ exceeds the initial shock $\left(\hat{K}_{0}>1\right)$ if $\underline{\beta} \gamma>1-\underline{\beta} \rho$. When this happens, $\hat{K}_{1}>0$ also. Note that we have for each $s$

$$
\hat{K}_{s}=\underline{\beta} \gamma^{s+1}\left(\frac{1}{1-\underline{\beta} \rho}\right)-\gamma\left(\frac{\gamma^{s}-\rho^{s}}{\gamma-\rho}\right) .
$$

Figure 3 plots the dynamics of capital and output for various levels of $\rho$. The higher is $\rho$, the larger is the initial boom (since the present value of the shock is larger), and the later is the eventual bust. However, for higher $\rho$ the bust is more prolonged because the reallocation of capital to the innovative sector lasts longer.

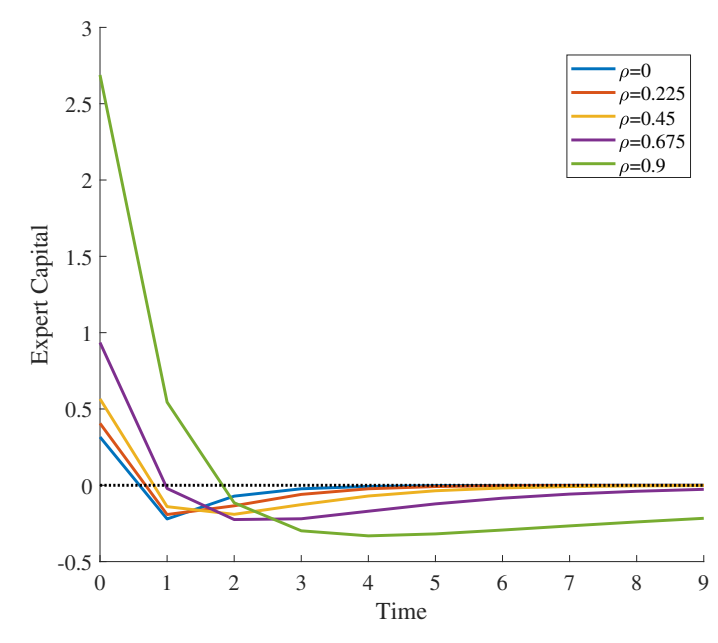

(a) Experts' Capital

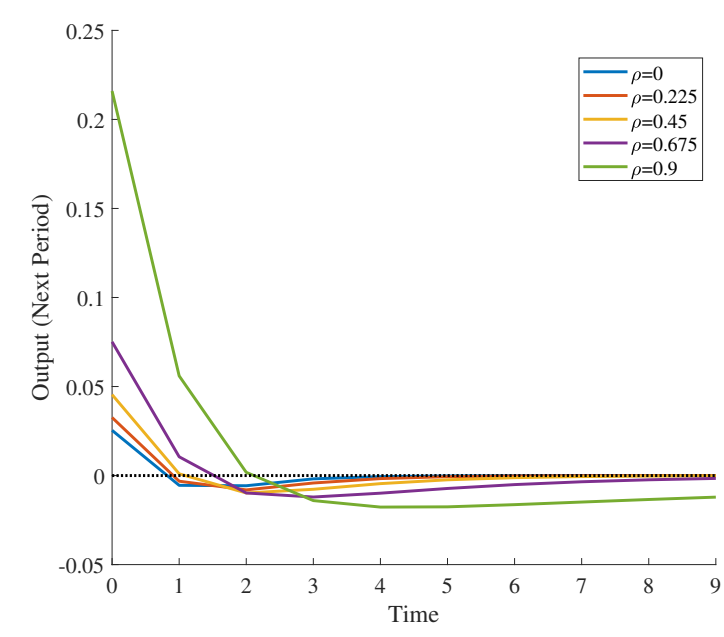

(b) Output

Figure 3: Changes in experts' capital $\hat{K}_{t}$, and output $\hat{Y}_{t+1}$ in response to news at $t=1$ decaying at rate $\rho$. 


\section{Discussion and Robustness}

The nature of our reallocative technology shock and the general equilibrium adjustments in credit markets are crucial for our story. We discuss these features in greater detail here.

\subsection{News, Noise, and Reallocation}

The shock we consider-news that primarily benefits agents other than the leveraged experts-is critical for our story. First, the economy does not experience boom-bust dynamics if the shock is immediate. If the innovative sector entered at $t=0$ (no news), then experts' capital holdings at $t=0$ would not change even though the asset price would immediately increase. In response to this contemporaneous shock, the asset price would increase at $t=0$, increasing experts' value of capital above the value of the debt they need to repay. This would increase experts' net worth, but since their increase in net worth is driven purely by an increase in the asset price, there would be no reallocation of capital. The value of experts' capital would increase, but that would not enable them to buy more capital. As a result, since experts' capital and debt were at steady state, the economy would immediately return to steady state without the innovative technology: $\hat{K}_{s}=0$ for all $s \geq 0$, and thus $\hat{q}_{s}=0$ for $s>0$. Experts would not take on any additional debt and thus there would be no contraction when the shock is gone. Hence, a shock without news would lead to a completely transitory expansion. Thus, news is critical to getting an expansion as well as a contraction after the shock.

Second, the boom-bust dynamics we describe are robust to whether or not the information is truly news or just "noise." We have modeled news about future innovative technology as a real technology shock that actually transpires, but all that matters for our story to get moving is positive expectations about future asset prices leading to a credit boom today. Thus, we could just as easily tell our story using a "behavioral shock," in which agents' expectations about the future increase, but perhaps in response to news that does not transpire. To see this, consider when agents receive news at $t$ of a technology shock at $t+1$, but the shock does not realize. Thus, agents enter the period with additional capital $\hat{K}_{t}$ and additional debt $\hat{K}_{t+1}+\hat{q}_{t+1}$, derived earlier. We denote the equilibrium prices and capital allocation going forward, once agents learn the shock does not in fact realize, by $\hat{q}$ and $\hat{K}$.

Proposition 4. Suppose the economy receive a news shock at $t=0$ regarding the productivity of the innovative sector at $t=1$, but at $t=1$ the shock does not transpire (i.e., $\hat{z}=0$ in fact). Then the economy experiences the following deterministic boom-bust dynamics from $t+1$ onward: 
1. Identical dynamics for experts capital for all periods: $\hat{K}_{s}=\hat{K}_{s}$ for all $s>0$; accordingly output dynamics are identical except at $t=1$ when output suffers (non-experts do not use the innovative technology),

2. A price crash at $t=1: \hat{q}_{1}=-\frac{r}{R \eta} \frac{(1-\underline{\beta} \gamma) \gamma \hat{z}}{1-\underline{\beta} \gamma}=-\frac{r \underline{\beta}}{1+\eta} \hat{z}<0$, whereas $\hat{q}_{1}>0$ if the shock occurs,

3. Capital prices going forward are identical $\hat{\hat{q}}_{s}=\hat{q}_{s}$ for all $s>1$.

In this case, if future asset prices are below the behavioral expectation, the economy would likely also feature defaults since collateral constraints would have been set on the expectation of higher collateral values making full debt enforcement impossible. Thus, behavioral assumptions would amplify our result. Bordalo et al. (2019) use Diagnostic expectations to examine the role of expectations in driving Minsky-type credit cycles with predictable returns but also predictable prediction errors.

Hence, our story that news ofa positive technology shock to an innovative sector produces boom-bust dynamics is very robust. Whether or not the technology shock realizes, we get the identical dynamics for capital for leveraged investors. Of course output and the asset price depend on whether the shock occurs or not. But as far as our story about endogenous cycles, once agents get the news, the cyclical properties of expert capital dynamics are already in motion. Whether the shock realizes later matters for some things at $t=1$, but either way the economy will experience a boom-bust cycle.

Importantly, that is not the case if news concerns the productivity of experts. Suppose instead that agents learn at $t=0$ that experts will be more productive at $t=1$. Then it really matters if the shock happens or not. Dynamics in this case are merely the main dynamics in Kiyotaki and Moore (1997) dampened by a factor $\beta$ since the shock occurs in the future: the experts' capital holding increase immediately, as does the capital price. However, the economy experiences a boom-bust cycle only if the shock doesn't occur at $t=1$ : agents expect the boom to continue at $t=1$ because experts will have lots of output from higher productivity to buy capital and repay their debts. But if productivity is not higher, then they cannot repay their (higher) debts and they are forced to sell capital. In this case, the cycle is not endogenous but the result of good information followed by bad information. In contrast, news about an innovative technology endogenously produces a cycle whether or not it is followed by "bad information" later. 


\subsection{Credit Frictions and Borrowing Constraints}

The general equilibrium costs of reallocation are crucial for a credit boom to expand beyond the sustainable level. This result is seen most clearly if we "assume away" the bust at $t=1$ : consider the pseudo-equilibrium in which we artificially set $\hat{K}_{1}=0$, implying also that $\hat{K}_{s}=\hat{q}_{s}=0$ for all $s>1$ as well. With this assumption, the pseudoequilibrium features a larger boom: $\hat{q}_{1}=r \underline{\beta} \hat{z}, \hat{q}_{0}=\frac{r \underline{\beta}^{2}}{\gamma} \hat{z}$, and $\hat{K}_{0}=\underline{\beta} \hat{z}$. Indeed, if $\underline{\beta}=1$, which would be a permissible parameterization in a finite-horizon model, then we have $\hat{K}_{0}=\hat{z}$, which would truly imply that $\hat{K}_{1}=0$, so that this pseudo-equilibrium would be the true equilibrium. In this case, experts can borrow the full change in future capital prices without changing the price of capital today. The demand for capital used for the innovative technology would increase the capital price by $\hat{z}$; experts would increase their capital holdings at $t=0$ by $\hat{z}$ and then at $t=1$ sell their capital for use in the innovative technology, returning their capital level to the steady-state. Thus, the economy would experience a boom at $t=0$ from higher expert capital, and also a boom at $t=1$ from the reallocation toward the innovative technology, and then return to steady state afterward. Because the economy features no "real rigidities" in this sense, the initial booms would not require costly reallocation leading to a bust in following periods.

One may worry that our results therefore depend critically on positive interest rates, but that is not the case. All that is required is some financial friction limiting borrowing to less than the full value of capital next period. For example, let the borrowing constraint be given by

$$
R b_{t}=\lambda_{t} q_{t+1} k_{t}
$$

where $\lambda_{t}<1$. The budget constraint for experts is now

$$
\left(q_{t}-\underline{\beta} \lambda_{t} q_{t+1}\right) K_{t}=a K_{t-1}+\left(1-\lambda_{t-1}\right) q_{t} K_{t-1}
$$

With a constant $\lambda$, steady-state values are as follows:

$$
q^{*}=\frac{a}{\lambda(1-\underline{\beta})}=\frac{R a}{\lambda r}, \quad u^{*}=\frac{a}{\lambda^{\prime}}
$$

where $u_{t}=q_{t}-\underline{\beta} q_{t+1}$ as before. As shown in Appendix C, the boom-bust dynamics go through with slight quantitative differences.

Including a tighter borrowing constraint allows us to emphasize the difference between technology shocks and a shock to the borrowing constraint, i.e. "financial shocks." We now consider shocks to credit markets directly, which we model as a temporary in- 
crease in $\lambda_{t}$. Let $\lambda_{0}=\lambda(1+\hat{\lambda})$ with $\hat{\lambda}>0$, and $\lambda_{s}=\lambda$ for $s>0$. Such a shock temporarily increases the flow of credit, reminiscient of a financial liberalization or expansion.

Proposition 5. In response to a shock regarding the collateral constraint, $\lambda_{0}=\lambda(1+\hat{\lambda})$ with $\hat{\lambda}>0$, and $\lambda_{s}=\lambda$ for $s>0$, the economy experiences the following deterministic dynamics:

1. A boom in expert capital at time $t=0: \hat{K}_{0}=\lambda \hat{\lambda}\left(\frac{R-\sigma-R \sigma / \eta^{\prime}}{r(R-\lambda)}\right)>0$,

2. A bust in expert capital going forward: $\hat{K}_{1}=\frac{\sigma \lambda}{R-\lambda}(\sigma-R) \hat{\lambda}<0$ and $\hat{K}_{s}<0$ for all $s>1$ returning to steady state at rate $\sigma$,

3. No change in capital price at $t=0$ but depressed prices going forward: $\hat{q}_{0}=0$ and $\hat{q}_{s}<0$ for all $s \geq 1$.

where $\sigma \equiv\left(\frac{1-\underline{\beta} \lambda}{1-\lambda \underline{\beta}+\lambda(1-\underline{\beta}) / \eta}\right)=\frac{1}{1+\frac{\lambda r}{(R-\lambda) \eta}}<1, \frac{\lambda r}{(R-\lambda)}<1, \sigma>\gamma$, and $\sigma \rightarrow \gamma$ as $\lambda \rightarrow 1$. Furthermore, if at $t=0$ agents learn that the collateral constraint shock will occur at $t=N$, then we have no dynamics until the shock occurs: $\hat{K}_{s}=\hat{q}_{s}=0$ for $s<N$, and then dynamics at $t=N$ are given as above with $\hat{q}_{N}=0$ and $\hat{K}_{N}=\lambda \hat{\lambda}\left(\frac{R-\sigma-R \sigma / \eta^{\prime}}{r(R-\lambda)}\right)$.

Thus, even though experts' capital holdings increase at $t=0$, the asset price does not change $\hat{q}_{0}=0$. Experts buy more capital because they can borrow more (the collateral constraint is relaxed). Capital prices fall going forward since $\hat{K}_{s}<0$ for $s>0$. By a similar exercise, the effect of $\hat{\lambda}$ in the future is quite similar, with an important twist. News of a future increase in $\lambda_{t}$ has absolutely no effect on equilibrium until the shock occurs. At that point, experts' capital holdings increase but the capital price does not, and then there is a bust (lower expert capital and capital prices) going forward.

The stylzed dynamics of a "Minsky Cycle" match dynamics caused by news of a reallocative technology shock, but not at all dynamics caused by a financial shock. Asset price booms are an important part of the Minsky narrative, but, perhaps surprisingly, a financial shock does not produce an asset boom at all, and instead the expansion of debt simply depresses future asset prices. Additionally, there is no role for news with a financial shock. The reallocative technology shock matches the Minsky narrative much better than a financial shock does. ${ }^{8}$

\footnotetext{
${ }^{8}$ An alternative potential way to model a shock directly to financial markets would be to consider a temporary change in the discount rate of non-experts, $\beta$. A temporary increase in the discount factor leads to an increase in the capital price, which relaxes borrowing constraints (if it occurs in the future) and increases experts' wealth (since they are leveraged). The equilibrium consequence of such a shock, whether the shock is immediate or in the future, is a persistent boom in expert capital holdings and the asset price. Thus, generating boom-bust dynamics requires a boom and bust in the shocks since a temporary shock to $\beta$ does not endogenously generate cycles.
} 


\section{Conclusion}

Major boom-bust cycles exhibit large positive productivity shocks followed by sharp, equally large reversals in productivity. We present a model in which news of a future productivity boom in an innovative sector relaxes borrowing constraints immediately, leading to a credit-filled boom. However, the expansion of credit is "not sustainable" and requires a contraction of credit when the innovative sector is most productive, leading to a slump in productivity going forward. These dynamics are more pronounced when information regards innovations in the far future. The predictable boom-bust cycles produced by reallocative technology shocks match the standard Minsky narrative in a way that shocks to financial markets directly do not.

Our results have important implications for welfare and policy. We have intentionally kept the model as simple and stripped-down as possible. Nonetheless, it is clear that the presence of collateral constraints create pecuniary externalities (see Dávila and Korinek, 2017). It is likely that, as is common in these models, the initial boom is inefficiently high, and welfare would improve if the initial boom, and therefore following bust, were smaller. Adding additional features such as nominal rigidities or the zero-lower bound, as other papers do in greater detail (see Rognlie et al., 2018; Farhi and Werning, 2020), would further exacerbate the welfare costs of the bust following the credit expansion. Either way, it is clear that the optimal policy is to mitigate the initial expansion to mitigate the size of the bust.

\section{References}

AKINCI, Ö. ANd R. Chahrour (2018): “Good news is bad news: Leverage cycles and sudden stops," Journal of International Economics, 114, 362-375. 1

Beaudry, P., D. Galizia, AND F. Portier (2017): "Is the macroeconomy locally unstable and why should we care?" NBER Macroeconomics Annual, 31, 479-530. 1, 1

(2018): "Reconciling Hayek's and Keynes' views of recessions," The Review of Economic Studies, 85, 119-156. 1

- (2020): "Putting the cycle back into business cycle analysis," American Economic Review, 110, 1-47. 1, 1

Bernanke, B. And M. Gertler (1989): "Agency Costs, Net Worth, and Business Fluctuations," The American Economic Review, 79, pp. 14-31. 1 
Bhattacharya, S., C. A. Goodhart, D. P. Tsomocos, and A. P. Vardoulakis (2015): “A reconsideration of Minsky's financial instability hypothesis," Journal of Money, Credit and Banking, 47, 931-973. 1

Blanchard, O. J., J.-P. L’Huillier, and G. Lorenzoni (2013): “News, noise, and fluctuations: An empirical exploration," American Economic Review, 103, 3045-70. 4.1

Bordalo, P., N. Gennaioli, A. Shleifer, and S. J. Terry (2019): “Real credit cycles," Harvard University. 5.1

Boz, E. (2009): "Can miracles lead to crises? the role of optimism in emerging markets crises," Journal of Money, Credit and Banking, 41, 1189-1215. 1

CaO, D. AND J.-P. L'Huillier (2018): “Technological revolutions and the three great slumps: A medium-run analysis," Journal of Monetary Economics, 96, 93-108. 1, 4.1

DÁvila, E. AND A. KorineK (2017): “Pecuniary Externalities in Economies with Financial Frictions," The Review of Economic Studies, rdxo1o. 6

Eggertsson, G. B. And P. Krugman (2012): “Debt, Deleveraging, and the Liquidity Trap: A Fisher-Minsky-Koo Approach," The Quarterly Journal of Economics, 127, 1469-1513. 1,1

FARHI, E. AND I. Werning (2020): “Taming a Minsky Cycle,” Tech. rep., MIT. 1, 6

FISHER, I. (1933): "The debt-deflation theory of great depressions," Econometrica: Journal of the Econometric Society, 337-357. 1

Gorton, G. And G. Ordonez (2020): "Good booms, bad booms," Journal of the European Economic Association, 18, 618-665. 1

Greenwood, R., S. G. Hanson, and L. J. Jin (2019): “Reflexivity in credit markets," Tech. rep., National Bureau of Economic Research. 1

Greenwood, R., S. G. Hanson, A. Shleifer, and J. A. Sørensen (2020): “Predictable Financial Crises," Tech. rep., Harvard Business School Working Paper. I

Gulen, H., M. Ion, ANd S. Rossi (2019): "Credit cycles, expectations, and corporate investment," Expectations, and Corporate Investment (April 9, 2019). I

KeYnes, J. M. (1936): The general theory of employment, interest, and money, Macmillan. I 
Kiyotaki, N. And J. Moore (1997): “Credit Cycles," Journal of Political Economy, 105, pp. 211-48. 1, 1, 2, 2.1, 2.3, 3.1, 6, 5.1

López-SAlido, D., J. C. Stein, ANd E. ZaKRaJšeK (2017): “Credit-market sentiment and the business cycle," The Quarterly Journal of Economics, 132, 1373-1426. 1

MinsKy, H. P. (1980): "Capitalist financial processes and the instability of capitalism," Journal of Economic Issues, 14, 505-523. (document)

(1982): "The financial-instability hypothesis: capitalist processes and the behavior of the economy," Hyman P. Minsky Archive. 282. I

(1986): Stabilizing an unstable economy, Yale University Press. I

Rognlie, M., A. Shleifer, AND A. Simsek (2018): "Investment hangover and the great recession," American Economic Journal: Macroeconomics, 10, 113-53. 1, 6

\section{A Proofs}

First, a lemma, which applies generally:

Lemma 6. If the capital price at $t$ can be written

$$
\hat{q}_{t}=\frac{r}{R \eta}\left(\frac{1}{1-\underline{\beta} \gamma} \hat{K}_{t}+X \eta\right),
$$

for some $X$. Then in equilibrium $\hat{K}_{t}=\frac{X(1-\underline{\beta} \gamma)}{1-\underline{\beta}}$.

Proof. Linearizing the budget constraint at $t$ yields $\hat{K}_{t}+\hat{u}_{t}=\frac{R}{r} \hat{q}_{t}$, which becomes

$$
\hat{K}_{t}(1+\eta)=\frac{R \eta}{r} \hat{q}_{t} \Longrightarrow\left(1+\frac{1}{\eta}\right) \hat{K}_{t}=\frac{R}{r} \hat{q}_{t}
$$

Plugging in the proposed capital price and solving for $\hat{K}_{t}$ yields the solution.

Proof of Proposition 1. Linearizing the expert's budget constraint at $t=0$ yields equation (19). Linearizing the experts' budget constraint in future periods yields $\hat{u}_{s+1}+\hat{K}_{s+1}=\hat{K}_{s}$ for $s>0$. At $t=1$ the user cost is given by $\hat{u}_{1}=\frac{1}{\eta} \hat{K}_{1}+\hat{z}$, and so we have

$$
\frac{1}{\eta} \hat{K}_{1}+\hat{z}+\hat{K}_{1}=\hat{K}_{0}, \Longrightarrow \quad \hat{K}_{1}=\gamma\left(\hat{K}_{0}-\hat{z}\right),
$$


where $\gamma \equiv 1 /\left(1+\frac{1}{\eta}\right)=\frac{\eta}{1+\eta}$ reflects the elasticity of non-expert demand for capital, and $\gamma<1$ since $\eta>0$. For $s>1$ the change in the user cost is determined entirely by capital holdings since there is no shock, and so

$$
\left(1+\frac{1}{\eta}\right) \hat{K}_{s}=\hat{K}_{s-1} \Longrightarrow \hat{K}_{s}=\gamma \hat{K}_{s-1} .
$$

Hence, for all $s \geq 1$ we have

$$
\hat{K}_{s}=\gamma^{s}\left(\hat{K}_{0}-\hat{z}\right) .
$$

From (12) we can write the capital price as

$$
\hat{q}_{0}=\frac{r}{R \eta} \sum_{s=0}^{\infty} \underline{\beta}^{s} \hat{K}_{s}+\underline{\beta} \frac{r}{R} \hat{z}
$$

where the $\hat{z}$ term reflects that the user cost at $t=1$ contains the shock. In order to plug in for $\hat{q}_{0}$, we execute the following manipulations:

$$
\begin{aligned}
\hat{q}_{0} & =\frac{r}{R \eta} \sum_{s=0}^{\infty} \underline{\beta}^{s} \gamma^{s}\left(\hat{K}_{0}-\hat{z}\right)+\frac{r}{R} \frac{1}{\eta} \hat{z}+\underline{\beta} \frac{r}{R} \hat{z}, \\
\frac{R}{r} \hat{q}_{0} & =\frac{1}{\eta}\left(\frac{1}{1-\underline{\beta} \gamma}\right)\left(\hat{K}_{0}-\hat{z}\right)+\hat{z}\left(\underline{\beta}+\frac{1}{\eta}\right), \\
\frac{R \eta}{r} \hat{q}_{0} & =\left(\frac{1}{1-\underline{\beta} \gamma}\right) \hat{K}_{0}-\hat{z}\left(\frac{1}{1-\underline{\beta} \gamma}-\underline{\beta} \eta-1\right) .
\end{aligned}
$$

Plugging in for $\hat{q}_{0}$ from the budget constraint, we have

$$
\begin{aligned}
(1+\eta) \hat{K}_{0} & =\left(\frac{1}{1-\underline{\beta} \gamma}\right) \hat{K}_{0}-\hat{z}\left(\frac{1}{1-\underline{\beta} \gamma}-\underline{\beta} \eta-1\right), \\
\hat{K}_{0}\left(\frac{(1+\eta)(1-\underline{\beta} \gamma)-1}{1-\underline{\beta} \gamma}\right) & =\hat{z}\left(\frac{(1+\underline{\beta} \eta)(1-\underline{\beta} \gamma)-1}{1-\underline{\beta} \gamma}\right), \\
\hat{K}_{0} & =\hat{z}\left(\frac{(1+\underline{\beta} \eta)(1-\underline{\beta} \gamma)-1}{(1+\eta)(1-\underline{\beta} \gamma)-1}\right), \\
\hat{K}_{0} & =\hat{z} \underline{\beta} \gamma .
\end{aligned}
$$

And so, $\hat{K}_{0}>0$. Similar calculations establish that $\hat{K}_{1}=-\hat{z} \gamma(1-\underline{\beta} \gamma)<0$. Thus, using (21), we also have $\hat{K}_{s}<0$ for all $s>0$. 
From the budget constraint at $t=0$, we have that the asset price is given by

$$
\frac{R}{r} \hat{q}_{0}=\left(1+\frac{1}{\eta}\right) \hat{z} \underline{\beta} \gamma \Longrightarrow \quad \hat{q}_{0}=r \underline{\beta}^{2} \hat{z}
$$

Since $\hat{K}_{s}<0$ for all $s>0$, it follows that $\hat{q}_{s}<0$ for all $s>0$.

Finally, we can write the capital price at $t=1$ as

$$
\hat{q}_{1}=\frac{r}{R}\left(\sum_{s=0}^{\infty} \underline{\beta^{s}} \frac{\hat{K}_{s+1}}{\eta}+\hat{z}\right)=\frac{r}{R}\left(\sum_{s=0}^{\infty}(\gamma \underline{\beta})^{s} \frac{\gamma\left(\hat{K}_{0}-\hat{z}\right)}{\eta}+\hat{z}\right),
$$

where $\hat{K}_{s}=\gamma^{s}\left(\hat{K}_{0}-\hat{z}\right)$. Taking the infinite sum, we have

$$
\begin{aligned}
\hat{q}_{1} & =\frac{r}{R}\left(\frac{\gamma}{\eta} \frac{\hat{K}_{0}-\hat{z}}{1-\underline{\beta} \gamma}+\hat{z}\right)=\frac{r}{R}\left(\frac{\gamma}{\eta} \frac{(\underline{\beta} \gamma \hat{z})-\hat{z}}{1-\underline{\beta} \gamma}+\hat{z}\right), \\
& =\frac{r}{R}\left(-\frac{\gamma}{\eta} \frac{\hat{z}(1-\underline{\beta} \gamma)}{1-\underline{\beta} \gamma}+\hat{z}\right)=\frac{r}{R}\left(-\frac{\gamma}{\eta} \hat{z}+\hat{z}\right), \\
& =r \underline{\beta} \gamma \hat{z},
\end{aligned}
$$

and hence $\hat{q}_{1}>0$. Equivalently, we can manipulate equation (19) by using $\hat{u}_{0}=\eta \hat{K}_{0}$ to write $(1+\eta) \hat{u}_{0}=\frac{R}{r} \hat{q}_{0}$. Plugging into the asset price equation $\hat{q}_{0}=\frac{r}{R} \hat{u}_{0}+\underline{\beta} \hat{q}_{1}$ we can write the recursion

$$
\hat{q}_{0}=\frac{1}{1+\eta} \hat{q}_{0}+\underline{\beta} \hat{q}_{1} \Longrightarrow \hat{q}_{1}=\frac{\gamma}{\underline{\beta}} \hat{q}_{0} .
$$

Note that again we have $\hat{q}_{1}=\frac{\gamma}{\underline{\beta}} r \underline{\beta^{2}} \hat{z}=r \underline{\beta} \gamma \hat{z}$.

Proof of Proposition 3. The key equations are the same as before, with the exception of the budget constraint and user cost at $t=N$ instead of at $t=1$. At time $t=N$, non-experts anticipate a higher marginal productivity of capital, so the user cost is given by

$$
\hat{u}_{N}=\frac{1}{\eta} \hat{K}_{N}+\hat{z},
$$

hence have

$$
\hat{K}_{N}=\gamma\left(\hat{K}_{N-1}-\hat{z}\right) .
$$

For $0 \leq s<N$,

$$
\hat{K}_{s}=\gamma^{s} \hat{K}_{0}
$$


and for $s \geq N$,

$$
\hat{K}_{s}=\gamma^{s} \hat{K}_{0}-\gamma^{s+1-N} \hat{z},
$$

Finally, since the capital price is the discounted sum of future user costs, we have

$$
\hat{q}_{0}=\frac{r}{R \eta} \sum_{s=0}^{\infty} \underline{\beta}^{s} \hat{K}_{s}+\underline{\beta}^{N} \frac{r}{R} \hat{z} .
$$

We then plug (24) and (25) into (26) and solve.

$$
\begin{aligned}
\hat{q}_{0} & =\frac{r}{R \eta} \sum_{s=0}^{\infty} \underline{\beta}^{s} \hat{K}_{s}+\underline{\beta}^{N} \frac{r}{R} \hat{z}, \\
\frac{R}{r} \hat{q}_{0} & =\frac{1}{\eta} \sum_{s=0}^{\infty} \underline{\beta}^{s} \gamma^{s} \hat{K}_{0}-\frac{1}{\eta} \sum_{s=N}^{\infty} \underline{\beta}^{s} \gamma^{s+1-N} \hat{z}+\underline{\beta}^{N} \hat{z}, \\
\frac{R \eta}{r} \hat{q}_{0} & =\frac{\hat{K}_{0}}{1-\underline{\beta} \gamma}+\frac{\hat{z}}{R^{N}}\left(-\frac{\gamma}{1-\underline{\beta} \gamma}+\eta\right), \frac{R \eta}{r} \\
\hat{q}_{0} & =\frac{\hat{K}_{0}}{1-\underline{\beta} \gamma}-\frac{\hat{z}}{R^{N-1}}\left(\frac{1}{1-\underline{\beta} \gamma}-\frac{\eta}{R}-1\right) .
\end{aligned}
$$

From Lemma 6 this implies

It then follows from $\frac{R}{r} \hat{q}_{0}=\left(1+\frac{1}{\eta}\right) \hat{K}_{0}$ that

$$
\hat{q}_{0}=r \underline{\beta}^{N+1} \hat{z} .
$$

From (25) we have

$$
\hat{K}_{N}=\gamma^{N} \hat{K}_{0}-\gamma \hat{z} \Longrightarrow \hat{K}_{N}=-\gamma \hat{z}\left(1-(\underline{\beta} \gamma)^{N}\right)<0 .
$$

Finally, we can write the capital price at $t=N$ as

$$
\hat{q}_{N}=\frac{r}{R}\left(\sum_{s=0}^{\infty} \underline{\beta}^{s} \frac{\hat{K}_{s+N}}{\eta}+\hat{z}\right)=\frac{r}{R}\left(\sum_{s=0}^{\infty}(\underline{\beta} \gamma)^{s} \frac{\hat{K}_{N}}{\eta}+\hat{z}\right) .
$$


We then use $\hat{K}_{N}=-\gamma \hat{z}\left(1-(\underline{\beta \gamma})^{N}\right)$. Taking the infinite sum, we have

$$
\begin{aligned}
\hat{q}_{N} & =\frac{r}{R}\left(-\frac{\hat{\gamma}}{\eta} \frac{\hat{z}\left(1-(\underline{\beta} \gamma)^{N}\right)}{1-\underline{\beta} \gamma}+\hat{z}\right)=r \underline{\beta} \hat{z}\left(-\frac{1}{1+\eta} \frac{\left(1-(\underline{\beta} \gamma)^{N}\right)}{1-\underline{\beta} \gamma}+1\right), \\
& =r \underline{\beta} \hat{z}\left(\frac{(\underline{\beta \gamma})^{N}-1}{1+\eta-\underline{\beta \eta}}+1\right)=r \underline{\beta} \hat{z}\left(\frac{(\underline{\beta \gamma})^{N}-1+1+\eta-\underline{\beta} \eta}{1+\eta-\underline{\beta} \eta}\right), \\
& =r \underline{\beta} \hat{z}\left(\frac{(\underline{\beta \gamma})^{N}+\eta-\underline{\beta \eta}}{1+\eta-\underline{\beta} \eta}\right)>0 .
\end{aligned}
$$

\section{B Persistent Shocks}

We first consider persistent decaying shocks (AR-1) and then a permanent shock.

\section{B.1 Persistent Shock Beginning at $t=1$}

Let's suppose that starting at $t=1$ the economy experiences an AR-1 decaying shock $\hat{z}_{s}=\rho^{s-1}$, with $\rho \in(0,1)$. We have $\hat{z}_{1}=1$ and then the shock decays at rate $\rho$ going forward. Accordingly, for $s>0$ we have the user cost

$$
\hat{u}_{s}=\frac{1}{\eta} \hat{K}_{s}+\hat{z}_{s}
$$

Capital dynamics are as follows. Linearizing budget constraints for $s>0$ we have

$$
\hat{K}_{s+1}=\gamma\left(\hat{K}_{s}-\hat{z}_{s+1}\right)
$$

We solve the model as before, plugging these conditions into the two key equations at $t=0$ : The budget constraint at is given by

$$
\hat{K}_{0}=\gamma \frac{R}{r} \hat{q}_{0}
$$


and the asset price is

$$
\hat{q}_{0}=\frac{r}{R} \sum_{s=0}^{\infty} \underline{\beta}^{s} \hat{u}_{s}=\frac{r}{R} \sum_{s=0}^{\infty} \underline{\beta}^{s}\left(\frac{1}{\eta} \hat{K}_{s}+\hat{z}_{s}\right),
$$

keeping in mind that $\hat{z}_{0}=0$.

Iterating forward the equation for capital dynamics, we have

$$
\hat{K}_{s}=\gamma^{s} \hat{K}_{0}-\sum_{i=1}^{s} \gamma^{s+1-i} \hat{z}_{i}=\gamma^{s} \hat{K}_{0}-\sum_{i=1}^{s} \gamma^{s+1-i} \rho^{i-1}=\gamma^{s} \hat{K}_{0}-\gamma^{s} \sum_{i=1}^{s}(\rho / \gamma)^{i-1}
$$

and summing yields

$$
\hat{K}_{s}=\gamma^{s} \hat{K}_{0}-\gamma^{s} \frac{1-\left(\frac{\rho}{\gamma}\right)^{s}}{1-\frac{\rho}{\gamma}}=\gamma^{s} \hat{K}_{t}-\gamma\left(\frac{\gamma^{s}-\rho^{s}}{\gamma-\rho}\right) .
$$

Plugging into the equation for the asset price, we have

$$
\begin{aligned}
\hat{q}_{0} & =\frac{r}{R}\left(\sum_{s=0}^{\infty} \underline{\beta}^{s}\left(\frac{1}{\eta} \hat{K}_{s}\right)+\sum_{s=1}^{\infty} \underline{\beta}^{s} \hat{z}_{s}\right), \\
& =\frac{r}{R}\left(\sum_{s=0}^{\infty} \underline{\beta}^{s} \frac{1}{\eta}\left(\gamma^{s} \hat{K}_{0}-\gamma\left(\frac{\gamma^{s}-\rho^{s}}{\gamma-\rho}\right)\right)+\sum_{s=1}^{\infty} \underline{\beta}^{s} \rho^{s-1}\right), \\
& =\frac{r}{R}\left(\frac{1}{\eta} \frac{1}{1-\underline{\beta} \gamma} \hat{K}_{0}\right)+\frac{r}{R}\left(\frac{\underline{\beta}}{1-\underline{\beta} \rho}\right)-\frac{r}{R} \frac{1}{\eta} \frac{\gamma}{\gamma-\rho}\left(\sum_{s=0}^{\infty}(\underline{\beta} \gamma)^{s}-(\underline{\beta} \rho)^{s}\right), \\
& =\frac{r}{R}\left(\frac{1}{\eta} \frac{1}{1-\underline{\beta} \gamma} \hat{K}_{0}\right)+\frac{r}{R}\left(\frac{\underline{\beta}}{1-\underline{\beta} \rho}\right)-\frac{r}{R} \frac{1}{\eta} \frac{\gamma}{\gamma-\rho}\left(\frac{1}{1-\underline{\beta} \gamma}-\frac{1}{1-\underline{\beta} \rho}\right), \\
& =\frac{r}{R}\left(\frac{1}{\eta} \frac{1}{1-\underline{\beta} \gamma} \hat{K}_{0}+\frac{\underline{\beta} \gamma(1-\underline{\beta})}{(1-\underline{\beta} \gamma)(1-\underline{\beta} \rho)}\right) .
\end{aligned}
$$

From Lemma 6 with $X=\frac{\underline{\beta} \gamma(1-\underline{\beta})}{(1-\underline{\beta} \gamma)(1-\underline{\beta} \rho)}$ we have

$$
\hat{K}_{0}=\underline{\beta} \gamma\left(\frac{1}{1-\underline{\beta} \rho}\right)>0 .
$$




\section{B.2 Persistent Shock Beginning $N$ Periods Forward, $t^{\prime}=N$}

Now suppose the shock starts at $t=N, \hat{z}_{s}=\rho^{s-N}$ for $s \geq N$ and $\hat{z}_{s}=0$ for $s<N$. Accordingly, for $s>0$ we have the user cost

$$
\hat{u}_{s}=\frac{1}{\eta} \hat{K}_{s}+\hat{z}_{s}
$$

Capital dynamics are as follows. Linearizing budget constraints for $s>0$ we have

$$
\hat{K}_{s+1}=\gamma\left(\hat{K}_{s}-\hat{z}_{s+1}\right),
$$

keeping in mind that the shock is zero for $s<N$.

We solve the model as before, plugging these conditions into the two key equations at $t=0$ : The budget constraint is given by

$$
\hat{K}_{0}=\gamma \frac{R}{r} \hat{q}_{0}
$$

and the asset price is

$$
\hat{q}_{0}=\frac{r}{R} \sum_{s=0}^{\infty} \underline{\beta}^{s} \hat{u}_{s}=\frac{r}{R} \sum_{s=0}^{\infty} \underline{\beta}^{s}\left(\frac{1}{\eta} \hat{K}_{s}+\hat{z}_{s}\right),
$$

keeping in mind that $\hat{z}_{s}=0$ for $s<N$.

Iterating forward the equation for capital dynamics, we have: for $0 \leq s<N, \hat{K}_{s}=$ $\gamma^{s} \hat{K}_{0}$ and then also

$$
\hat{K}_{N+s}=\gamma^{s+N} \hat{K}_{0}-\sum_{i=0}^{s} \gamma^{s+1-i} \hat{z}_{i}=\gamma^{s+N} \hat{K}_{0}-\sum_{i=0}^{s} \gamma^{s+1-i} \rho^{i}=\gamma^{s+N} \hat{K}_{0}-\gamma^{s+1} \sum_{i=0}^{s}(\rho / \gamma)^{i} .
$$

Summing yields

$$
\hat{K}_{N+s}=\gamma^{s+N} \hat{K}_{0}-\gamma^{s+1} \frac{1-\left(\frac{\rho}{\gamma}\right)^{s+1}}{1-\frac{\rho}{\gamma}}=\gamma^{s+N} \hat{K}_{0}-\gamma\left(\frac{\gamma^{s+1}-\rho^{s+1}}{\gamma-\rho}\right),
$$

implying for $s \geq N$ we can write

$$
\hat{K}_{s}=\gamma^{s} \hat{K}_{0}-\gamma\left(\frac{\gamma^{s+1-N}-\rho^{s+1-N}}{\gamma-\rho}\right),
$$


Plugging into the equation for the asset price, starting the shock at $t=N$, we have

$$
\begin{aligned}
\hat{q}_{0} & =\frac{r}{R}\left(\sum_{s=0}^{\infty} \underline{\beta}^{s}\left(\frac{1}{\eta} \hat{K}_{s}\right)+\sum_{s=N}^{\infty} \underline{\beta}^{s} \hat{z}_{s}\right), \\
& =\frac{r}{R}\left(\sum_{s=0}^{\infty} \underline{\beta}^{s} \frac{1}{\eta} \gamma^{s} \hat{K}_{0}+\sum_{s=N}^{\infty} \underline{\beta}^{s}\left(\rho^{s-N}-\frac{\gamma}{\eta}\left(\frac{\gamma^{s+1-N}-\rho^{s+1-N}}{\gamma-\rho}\right)\right)\right), \\
& =\frac{r}{R}\left(\frac{1}{\eta} \frac{1}{1-\underline{\beta} \gamma} \hat{K}_{0}\right)+\frac{r}{R}\left(\frac{\underline{\beta}^{N}}{1-\underline{\beta} \rho}\right)-\frac{r}{R} \frac{1}{\eta} \frac{\gamma}{\gamma-\rho}\left(\sum_{s=N}^{\infty} \gamma^{1-N}(\underline{\beta} \gamma)^{s}-\rho^{1-N}(\underline{\beta} \rho)^{s}\right), \\
& =\frac{r}{R}\left(\frac{1}{\eta} \frac{1}{1-\underline{\beta} \gamma} \hat{K}_{0}\right)+\frac{r}{R}\left(\frac{\underline{\beta}^{N}}{1-\underline{\beta} \rho}\right)-\frac{r}{R} \frac{1}{\eta} \frac{\gamma}{\gamma-\rho}\left(\frac{\underline{\beta}^{N} \gamma}{1-\underline{\beta} \gamma}-\frac{\underline{\beta}^{N} \rho}{1-\underline{\beta} \rho}\right), \\
& =\frac{r}{R}\left(\frac{1}{\eta} \frac{1}{1-\underline{\beta} \gamma} \hat{K}_{0}+\frac{\underline{\beta}^{N} \gamma(1-\underline{\beta})}{(1-\underline{\beta} \gamma)(1-\underline{\beta} \rho)}\right) .
\end{aligned}
$$

Note that we now have $X=\frac{\underline{\beta}^{N} \gamma(1-\underline{\beta})}{(1-\underline{\beta} \gamma)(1-\underline{\beta} \rho)}$. From Lemma 6 we have

$$
\hat{K}_{0}=\underline{\beta}^{N} \gamma\left(\frac{1}{1-\underline{\beta} \rho}\right)>0 .
$$

Figures 4 and 5 plots the dynamics of capital and output, varying $N=1, \ldots, 5$ for $\rho=0.5$ and $\rho=0.9$.

\section{B.3 Permanent Shock}

Suppose that the shock $\hat{z}$ occurs in every period after $t=1$. The key equations are

$$
\hat{K}_{0}=\gamma \frac{R}{r} \hat{q}_{0}, \quad \hat{q}_{0}=\frac{r}{R} \sum_{s=0}^{\infty} \underline{\beta^{s}} \hat{u}_{s}, \quad \hat{K}_{s+1}=\gamma\left(\hat{K}_{s}-\hat{z}\right) .
$$

Note that the last equation implies that

$$
\hat{K}_{N}=\gamma^{N} \hat{K}_{0}-\hat{z} \sum_{s=1}^{N} \gamma^{s}=\gamma^{N} \hat{K}_{0}-\hat{z} \frac{\gamma-\gamma^{N+1}}{1-\gamma} .
$$

For $s>0$ we have

$$
\hat{u}_{s}=\frac{1}{\eta} \hat{K}_{s}+\hat{z}
$$




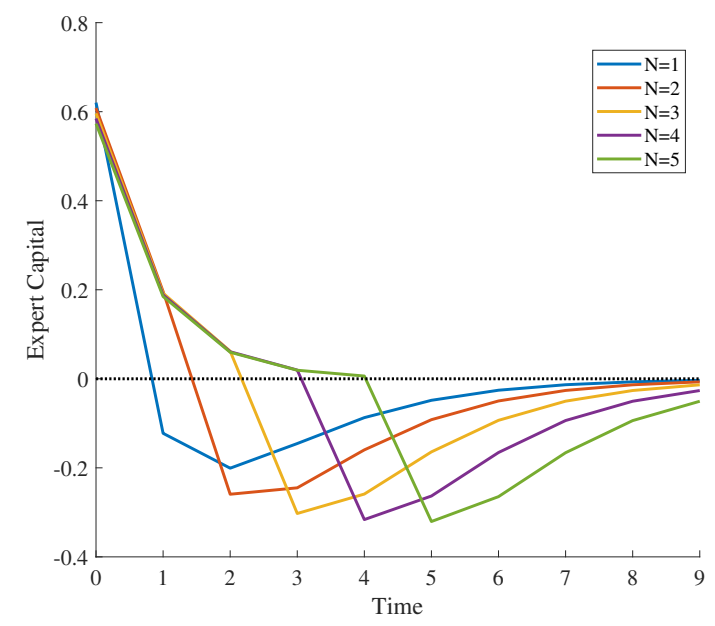

(a) Experts' Capital $\hat{K}_{t}$

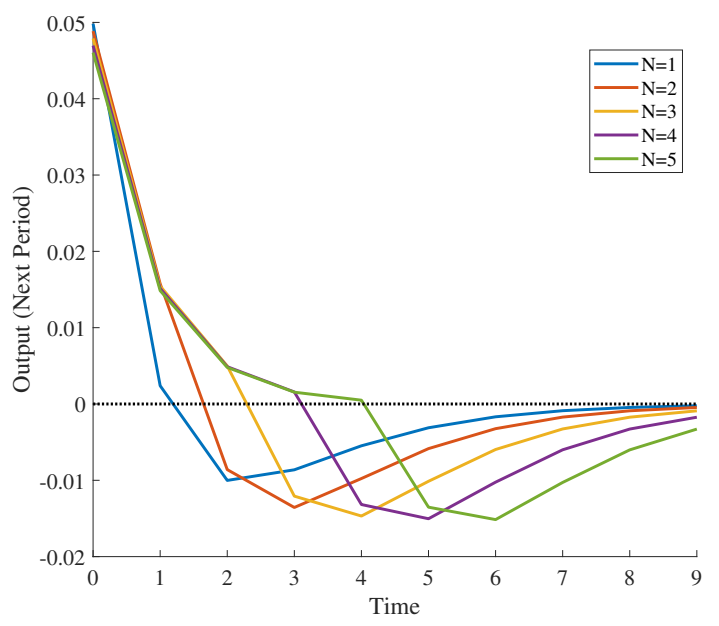

(b) Output next period $\hat{Y}_{t+1}$

Figure 4: Changes in experts' capital and output in response to news at $t=N$, varying $N$, decaying at rate $\rho=0.5$.

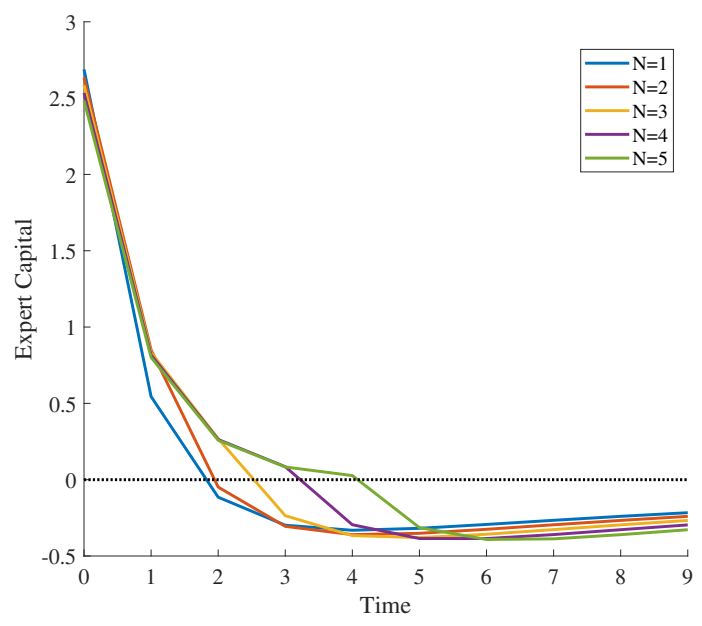

(a) Experts' Capital $\hat{K}_{t}$

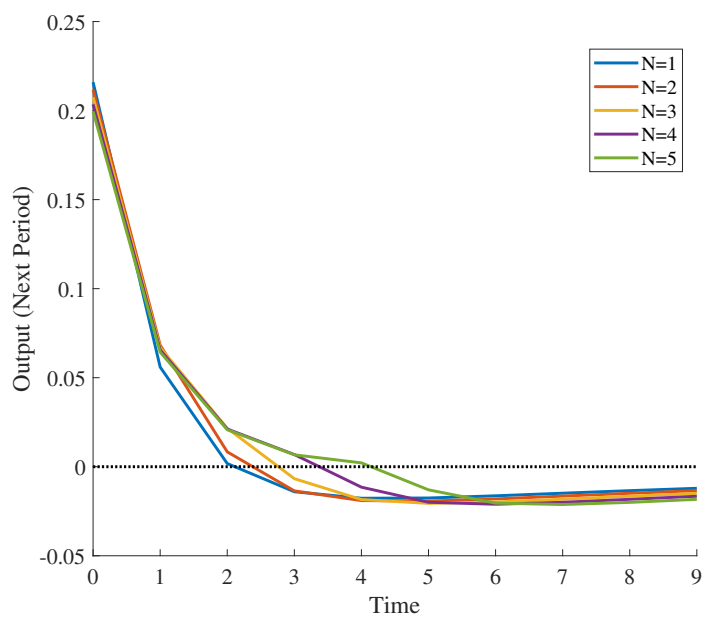

(b) Output next period $\hat{Y}_{t+1}$

Figure 5: Changes in experts' capital and output in response to news at $t=N$, varying $N$, decaying at rate $\rho=0.9$.

and so the asset price can be written

$$
\begin{aligned}
\hat{q}_{0} & =\frac{r}{R}\left(\sum_{s=0}^{\infty} \underline{\beta}^{s}\left(\frac{1}{\eta} \hat{K}_{s}\right)+\sum_{s=1}^{\infty} \underline{\beta}^{s} \hat{z}\right), \\
& =\frac{r}{R}\left(\sum_{s=0}^{\infty} \underline{\beta}^{s}\left(\frac{1}{\eta} \gamma^{s} \hat{K}_{0}\right)+\sum_{s=1}^{\infty} \underline{\beta}^{s}\left(-\hat{z} \frac{1}{\eta} \frac{\gamma-\gamma^{s+1}}{1-\gamma}+\hat{z}\right)\right), \\
& =\frac{r}{R}\left(\frac{1}{\eta} \frac{1}{1-\underline{\beta} \gamma} \hat{K}_{0}+\hat{z} \frac{\underline{\beta \gamma}}{1-\underline{\beta \gamma}}\right) .
\end{aligned}
$$


From Lemma 6 we have

$$
\hat{K}_{0}=\hat{z} \frac{\underline{\beta} \gamma}{1-\underline{\beta}}=\hat{z} \frac{\gamma}{r}>0
$$

Note that asymptotically $\hat{K}_{s} \rightarrow-\hat{z} \frac{\gamma}{1-\gamma}=-\eta \hat{z}$. We converge back to the original steady state price $q^{*}$, but first the price rises and experts hold more capital because collateral constraints are relaxed. But the price converges back to the steady state, and experts hold less capital, consistent with non-experts' increased productivity.

\section{Tight Borrowing Constraints}

Let the borrowing constraint be given by

$$
R b_{t}=\lambda_{t} q_{t+1} k_{t}
$$

where $\lambda_{t}<1$. The budget constraint for experts is now

$$
\left(q_{t}-\underline{\beta} \lambda_{t} q_{t+1}\right) K_{t}=a K_{t-1}+\left(1-\lambda_{t-1}\right) q_{t} K_{t-1}
$$

With a constant $\lambda$, steady-state values are as follows:

$$
q^{*}=\frac{a}{\lambda(1-\underline{\beta})}=\frac{R a}{\lambda r}, \quad u^{*}=\frac{a}{\lambda}
$$

where $u_{t}=q_{t}-\underline{\beta} q_{t+1}$ as before.

We first reconsider the main results in the paper, which are quantitatively dampened if $\lambda<1$ but otherwise the same, and then consider shocks to $\lambda_{t}$. We refer to shocks to $\lambda_{t}$ as "financial shocks." Our main findings are that the consequences of financial shocks are quite distinct from the consequences of technology shocks.

\section{C.1 Technology Shocks}

We first consider a technology shock $\hat{z}$ as before.

Proposition 7. In response to a news shock at $t=0$ regarding the productivity of the innovative sector at $t=1$, the economy experiences the following deterministic boom-bust dynamics:

1. A larger increase in capital prices at $t=1: \hat{q}_{1}=r \sigma \underline{\beta} \hat{z}>r \gamma \underline{\beta} \hat{z}$, 
2. A dampened boom at time $t=0: \hat{K}_{0}=\underline{\beta}\left(\frac{\eta}{1+\left(\frac{R-\lambda}{r \lambda}\right) \eta}\right) \hat{z}<\underline{\beta} \gamma \hat{z}$, and $\hat{q}_{0}=r \underline{\beta}^{2} \hat{z}>0$ (same),

3. A dampened but prolonged bust going forward: $\hat{K}_{1}=-\sigma\left(\frac{r \lambda}{R-\lambda}\right)(1-\underline{\beta \sigma}) \hat{z}>-\gamma(1-$ $\underline{\beta \gamma}) \hat{z}$, and $\hat{K}_{s}=\sigma^{s}\left(\hat{K}_{t+s-1}-\hat{z}\left(\frac{\lambda r}{R-\lambda}\right)\right)<0$ for all $s \geq 1$, and $\hat{q}_{s+1}<0$ for all $s \geq 1$, where $\sigma \equiv\left(\frac{1-\underline{\beta} \lambda}{1-\lambda \underline{\beta}+\lambda(1-\underline{\beta}) / \eta}\right)=\frac{1}{1+\frac{\lambda r}{(R-\lambda) \eta}}<1, \frac{\lambda r}{(R-\lambda)}<1, \sigma>\gamma$, and $\sigma \rightarrow \gamma$ as $\lambda \rightarrow 1$.

The tighter borrowing constraint has two consequences for dynamics. First, the initial response is dampened because experts are less leveraged and thus credit markets have less of a role in propagating shocks. The result extends analogously when considering news $N$ periods forward, multiplying the initial capital deviations by $\beta^{N}$ as in the main model. Second, deviations from steady state are more persistent $(\sigma>\gamma)$ and so it takes longer to recover from the bust. However, the bust is not so severe.

Proof of Proposition 7. We first log-linearize the budget constraint at $t=s$ when there is no technology shock. In this case, debt is set with perfect foresight and we have

$$
\hat{K}_{s}=\sigma \hat{K}_{s-1}
$$

where $\sigma \equiv\left(\frac{1-\underline{\beta} \lambda}{1-\lambda \underline{\beta}+\lambda(1-\underline{\beta}) / \eta}\right)=\frac{1}{1+\frac{\lambda r}{(R-\lambda) \eta}}<1$. We can define $\eta^{\prime} \equiv \frac{(R-\lambda) \eta}{r \lambda}$ and then we have $\sigma=\frac{1}{1+\frac{1}{\eta^{\prime}}}=\frac{\eta^{\prime}}{1+\eta^{\prime}}$, analogous to the definition of $\gamma$. Note that $\sigma \rightarrow \gamma$ as $\lambda \rightarrow 1$ and that $\sigma>\gamma$ since $\frac{\lambda r}{(R-\lambda)}<1$.

In the period with the technology shock we instead would have

$$
\begin{aligned}
& \lambda(1-\underline{\beta})\left(\hat{K}_{s} / \eta+\hat{z}\right)+\hat{K}_{s}(1-\lambda \underline{\beta})=\hat{K}_{s-1}(\lambda(1-\underline{\beta})+1-\lambda), \\
& \hat{K}_{s}(1-\lambda \underline{\beta}+\lambda(1-\underline{\beta}) / \eta)=\hat{K}_{s-1}(1-\underline{\beta} \lambda)-\lambda(1-\underline{\beta}) \hat{z}, \\
& \hat{K}_{s}=\hat{K}_{s-1}\left(\frac{1-\underline{\beta} \lambda}{1-\lambda \underline{\beta}+\lambda(1-\underline{\beta}) / \eta}\right)-\hat{z}\left(\frac{\lambda(1-\underline{\beta})}{1-\lambda \underline{\beta}+\lambda(1-\underline{\beta}) / \eta}\right),
\end{aligned}
$$

which we can write as

$$
\hat{K}_{s}=\sigma\left(\hat{K}_{s-1}-\hat{z}\left(\frac{\lambda(1-\underline{\beta})}{1-\lambda \underline{\beta}}\right)\right)=\sigma\left(\hat{K}_{s-1}-\hat{z}\left(\frac{\lambda r}{R-\lambda}\right)\right) .
$$

Since $\frac{\lambda r}{R-\lambda}<1$, it is as if the shock enters in a smaller way compared to the baseline model (i.e., with $\lambda=1$ ). 
Finally, we log-linearize the budget constraint at $t=0$. We can write the budget constraint as

$$
\hat{K}_{0}(1-\underline{\beta} \lambda+\lambda(1-\underline{\beta}) / \eta)=\lambda \hat{q}_{0}
$$

which we can write as

$$
\hat{K}_{0}=\sigma \frac{\lambda}{1-\underline{\beta} \lambda} \hat{q}_{0}
$$

or equivalently,

$$
\hat{K}_{0}\left(1+\frac{(R-\lambda) \eta}{r \lambda}\right)=\hat{K}_{0}\left(1+\eta^{\prime}\right)=\frac{R \eta}{r} \hat{q}_{0}
$$

With the shock occurring at $t=1$, for all $s \geq 1$ we have

$$
\hat{K}_{s}=\sigma^{s}\left(\hat{K}_{s-1}-\hat{z}\left(\frac{\lambda(1-\underline{\beta})}{1-\lambda \underline{\beta}}\right)\right)=\sigma^{s}\left(\hat{K}_{s-1}-\hat{z}\left(\frac{\lambda r}{R-\lambda}\right)\right) .
$$

From (12) we can write the capital price as

$$
\hat{q}_{0}=\frac{1-\underline{\beta}}{\eta} \sum_{s=0}^{\infty} \underline{\beta}^{s} \hat{K}_{s}+\underline{\beta}(1-\underline{\beta}) \hat{z},
$$

where the $\hat{z}$ term reflects that the user cost at $t=1$ contains the shock. In order to plug in for $\hat{q}_{0}$, we execute the following manipulations:

$$
\begin{aligned}
\hat{q}_{0} & =\frac{r}{R \eta} \sum_{s=0}^{\infty} \underline{\beta}^{s} \sigma^{s}\left(\hat{K}_{0}-\hat{z}\left(\frac{\lambda(1-\underline{\beta})}{1-\lambda \underline{\beta}}\right)\right)+\frac{r}{R} \frac{1}{\eta} \hat{z}\left(\frac{\lambda(1-\underline{\beta})}{1-\lambda \underline{\beta}}\right)+\underline{\beta} \frac{r}{R} \hat{z}, \\
\frac{R}{r} \hat{q}_{0} & =\frac{1}{\eta}\left(\frac{1}{1-\underline{\beta} \sigma}\right)\left(\hat{K}_{0}-\hat{z}\left(\frac{\lambda(1-\underline{\beta})}{1-\lambda \underline{\beta}}\right)\right)+\hat{z}\left(\underline{\beta}+\frac{1}{\eta}\left(\frac{\lambda(1-\underline{\beta})}{1-\lambda \underline{\beta}}\right)\right), \\
\frac{R \eta}{r} \hat{q}_{0} & =\left(\frac{1}{1-\underline{\beta} \sigma}\right) \hat{K}_{0}-\hat{z}\left(\frac{1}{1-\underline{\beta} \sigma}\left(\frac{\lambda(1-\underline{\beta})}{1-\lambda \underline{\beta}}\right)-\underline{\beta} \eta-\left(\frac{\lambda(1-\underline{\beta})}{1-\lambda \underline{\beta}}\right)\right), \\
\frac{R \eta}{r} \hat{q}_{0} & =\left(\frac{1}{1-\underline{\beta \sigma}}\right) \hat{K}_{0}-\hat{z} \underline{\beta}\left(\frac{\sigma}{1-\underline{\beta \sigma}}\left(\frac{\lambda(1-\underline{\beta})}{1-\lambda \underline{\beta}}\right)-\eta\right), \\
\frac{R \eta}{r} \hat{q}_{0} & =\left(\frac{1}{1-\underline{\beta \sigma} \sigma}\right) \hat{K}_{0}-\hat{z} \underline{\beta}\left(\frac{\sigma}{1-\underline{\beta \sigma} \sigma}\left(\frac{r \lambda}{R-\lambda}\right)-\eta\right), \\
\frac{R \eta}{r} \hat{q}_{0} & =\left(\frac{1}{1-\underline{\beta} \sigma}\right) \hat{K}_{0}-\hat{z}\left(\frac{r \lambda}{R-\lambda}\right) \underline{\beta}\left(\frac{\sigma}{1-\underline{\beta} \sigma}-\eta\left(\frac{R-\lambda}{r \lambda}\right)\right) .
\end{aligned}
$$


Note that we can write the budget constraint in equation (37) as

$$
\hat{K}_{0}\left(1+\eta^{\prime}\right)=\frac{R \eta}{r} \hat{q}_{0}
$$

Hence we can write

$$
\hat{K}_{0}\left(1+\eta^{\prime}\right)=\left(\frac{1}{1-\underline{\beta \sigma} \sigma}\right) \hat{K}_{0}-\hat{z}\left(\frac{r \lambda}{R-\lambda}\right) \underline{\beta}\left(\frac{\sigma}{1-\underline{\beta} \sigma}-\eta\left(\frac{R-\lambda}{r \lambda}\right)\right),
$$

which is identical to the result from earlier with $\eta^{\prime}$ replacing $\eta, \sigma$ replacing $\gamma$, and $\hat{z}$ multiplied by $\left(\frac{r \lambda}{R-\lambda}\right)$. Since $\sigma=\frac{1}{1+1 / \eta^{\prime}}$, we can therefore solve out to get

$$
\hat{K}_{0}=\underline{\beta} \sigma \hat{z}\left(\frac{r \lambda}{R-\lambda}\right)=\underline{\beta}\left(\frac{\eta}{1+\left(\frac{R-\lambda}{r \lambda}\right) \eta}\right) \hat{z}<\underline{\beta} \gamma \hat{z}
$$

where the final inequality follows because $\frac{R-\lambda}{r \lambda}>1$ and $\gamma=\frac{\eta}{1+\eta}$. From the budget constraint we have

$$
\frac{R \eta}{r} \hat{q}_{0}=\hat{K}_{0}\left(1+\eta^{\prime}\right) \Longrightarrow \hat{q}_{0}=r \underline{\beta}^{2} \hat{z} .
$$

Plugging $\hat{K}_{0}\left(1+\eta^{\prime}\right)=\frac{R \eta}{r} \hat{q}_{0}$ into the asset price equation $\hat{q}_{0}=\frac{r}{R} \hat{u}_{0}+\beta \hat{q}_{1}$ we can write the recursion

$$
\hat{q}_{0}=\frac{1}{1+\eta^{\prime}} \hat{q}_{0}+\underline{\beta} \hat{q}_{1} \Longrightarrow \hat{q}_{1}=\frac{\sigma}{\underline{\beta}} \hat{q}_{0} .
$$

Note that we have $\hat{q}_{1}=\frac{\sigma}{\beta} r \underline{\beta}^{2} \hat{z}=r \underline{\beta} \sigma \hat{z}$.

Additionally, we have

$$
\hat{K}_{1}=-\sigma\left(\frac{r \lambda}{R-\lambda}\right)(1-\underline{\beta} \sigma) \hat{z}>-\gamma(1-\underline{\beta} \gamma) \hat{z},
$$

which is closer to zero than we get when $\lambda=1$.

\section{C.2 Financial Shocks Proof}

Proof of Proposition 5. Log-linearizing the budget constraint at $t=0$, we have

$$
q^{*} K^{*}\left(\hat{q}_{0}+\hat{K}_{0}\right)-\underline{\beta} \lambda q^{*} K^{*}\left(\hat{q}_{1}+\hat{K}_{0}+\hat{\lambda}_{0}\right)=1 q^{*} \hat{q}_{0} K^{*},
$$


where the RHS reflects that debt equals $\lambda q^{*} K^{*}$ and capital is predetermined. Rearranging and collecting terms we have

$$
\begin{aligned}
\left(\hat{q}_{0}+\hat{K}_{0}\right)-\underline{\beta} \lambda\left(\hat{q}_{1}+\hat{K}_{0}+\hat{\lambda}_{0}\right) & =\hat{q}_{0} \\
\lambda \hat{q}_{0}-\underline{\beta} \lambda \hat{q}_{1}+\hat{K}_{0}(1-\underline{\beta} \lambda) & =\lambda \hat{q}_{0}+\underline{\beta} \lambda \hat{\lambda}_{0} \\
\hat{K}_{0}(1-\underline{\beta} \lambda+\lambda(1-\underline{\beta}) / \eta) & =\lambda \hat{q}_{0}+\underline{\beta} \lambda \hat{\lambda}_{0}
\end{aligned}
$$

which we can write as

$$
\hat{K}_{0}\left(1+\eta^{\prime}\right)=\frac{R \eta}{r} \hat{q}_{0}+\frac{\eta}{r} \hat{\lambda}_{0}
$$

Next, consider the budget constraint at $t=1$. In this case, debt is set with perfect foresight and we have

$$
\begin{aligned}
q^{*} K^{*}\left(\hat{q}_{1}+\hat{K}_{1}\right)-\underline{\beta} \lambda q^{*} K^{*}\left(\hat{q}_{2}+\hat{K}_{1}\right) & =a K^{*} \hat{K}_{0}+q^{*} K^{*}\left(\hat{q}_{1}+\hat{K}_{0}\right)-\lambda q^{*} K^{*}\left(\hat{q}_{1}+\hat{K}_{0}+\hat{\lambda}\right), \\
\left(\hat{q}_{1}+\hat{K}_{1}\right)-\underline{\beta} \lambda\left(\hat{q}_{2}+\hat{K}_{1}\right) & =\lambda(1-\underline{\beta}) \hat{K}_{0}+(1-\lambda)\left(\hat{q}_{1}+\hat{K}_{0}\right)-\lambda \hat{\lambda}, \\
\lambda \hat{q}_{1}-\underline{\beta} \lambda \hat{q}_{2}+\hat{K}_{1}(1-\lambda \underline{\beta}) & =\lambda(1-\underline{\beta}) \hat{K}_{0}+(1-\lambda) \hat{K}_{0}-\lambda \hat{\lambda}, \\
\lambda(1-\underline{\beta}) \hat{K}_{1} / \eta+\hat{K}_{1}(1-\lambda \underline{\beta}) & =\hat{K}_{0}(\lambda(1-\underline{\beta})+1-\lambda)-\lambda \hat{\lambda}, \\
\hat{K}_{1}(1-\lambda \underline{\beta}+\lambda(1-\underline{\beta}) / \eta) & =\hat{K}_{0}(1-\underline{\beta} \lambda)-\lambda \hat{\lambda},
\end{aligned}
$$

which we can write as

$$
\hat{K}_{1}=\hat{K}_{0}\left(\frac{1-\underline{\beta} \lambda}{1-\lambda \underline{\beta}+\lambda(1-\underline{\beta}) / \eta}\right)-\frac{\lambda}{1-\underline{\beta} \lambda}\left(\frac{1-\underline{\beta} \lambda}{1-\lambda \underline{\beta}+\lambda(1-\underline{\beta}) / \eta}\right) \hat{\lambda},
$$

or equivalently

$$
\hat{K}_{1}=\sigma\left(\hat{K}_{0}-\frac{\lambda}{1-\underline{\beta} \lambda} \hat{\lambda}\right)=\sigma\left(\hat{K}_{0}-\frac{R \lambda}{R-\lambda} \hat{\lambda}\right) .
$$

Then equation (34) holds in every period thereafter, $\hat{K}_{s}=\sigma \hat{K}_{s-1}=\sigma^{s}\left(\hat{K}_{0}-\frac{\lambda}{1-\underline{\beta} \lambda} \hat{\lambda}\right)$.

From (12) we can write the capital price as $\hat{q}_{0}=\frac{r}{R \eta} \sum_{s=0}^{\infty} \underline{\beta}^{s} \hat{K}_{s}$. Then we have

$$
\begin{aligned}
\hat{q}_{0} & =\frac{r}{R \eta} \hat{K}_{0}+\frac{r}{R \eta} \sum_{s=1}^{\infty} \underline{\beta}^{s} \sigma^{s}\left(\hat{K}_{0}-\frac{\lambda}{1-\underline{\beta} \lambda} \hat{\lambda}\right), \\
\frac{R \eta}{r} \hat{q}_{0} & =\left(\frac{1}{1-\underline{\beta \sigma} \sigma}\right) \hat{K}_{0}-\hat{\lambda}\left(\frac{\underline{\beta} \sigma}{1-\underline{\beta} \sigma}\right)\left(\frac{\lambda}{1-\underline{\beta} \lambda}\right) .
\end{aligned}
$$


Plugging into the budget constraint we have

$$
\begin{aligned}
\left(1+\eta^{\prime}\right) \hat{K}_{0} & =\left(\frac{1}{1-\underline{\beta} \sigma}\right) \hat{K}_{t}-\hat{\lambda}\left(\frac{\underline{\beta} \sigma}{1-\underline{\beta} \sigma}\right)\left(\frac{\lambda}{1-\underline{\beta} \lambda}\right)+\frac{\eta}{r} \hat{\lambda}_{0} \\
\hat{K}_{0}\left(\left(1+\eta^{\prime}\right)(1-\underline{\beta} \sigma)-1\right) & =\hat{\lambda}\left(\frac{\eta}{r}(1-\underline{\beta} \sigma)-(\underline{\beta \sigma})\left(\frac{\lambda}{1-\underline{\beta} \lambda}\right)\right) \\
\hat{K}_{0}\left(\eta^{\prime}(1-\underline{\beta})\right) & =\hat{\lambda}\left(\frac{\eta}{r}(1-\beta \sigma)-\left(\frac{\underline{\beta} \sigma \lambda}{1-\underline{\beta} \lambda}\right)\right) \\
\hat{K}_{0}\left(\eta^{\prime}(1-\underline{\beta})\right) & =\hat{\lambda}\left(\frac{\eta^{\prime} \lambda}{R-\lambda}(1-\beta \sigma)-\left(\frac{\sigma \lambda}{R-\lambda}\right)\right) \\
\hat{K}_{0}\left(\eta^{\prime}(1-\underline{\beta})\right) & =\hat{\lambda}\left(\frac{\eta^{\prime} \lambda(1-\beta \sigma)-\lambda \sigma}{R-\lambda}\right) \\
\hat{K}_{0} & =\hat{\lambda}\left(\frac{\eta^{\prime} \lambda(1-\beta \sigma)-\lambda \sigma}{\eta^{\prime}(1-\underline{\beta})(R-\lambda)}\right) \\
\hat{K}_{0} & =\lambda \hat{\lambda}\left(\frac{1-\beta \sigma-\sigma / \eta^{\prime}}{(1-\underline{\beta})(R-\lambda)}\right) \\
\hat{K}_{0} & =\lambda \hat{\lambda}\left(\frac{R-\sigma-R \sigma / \eta^{\prime}}{r(R-\lambda)}\right) \\
\hat{K}_{0} & =\hat{\lambda}\left(\frac{\sigma \lambda}{R-\lambda}\right) .
\end{aligned}
$$

Note that this implies that

$$
\hat{K}_{1}=\sigma\left(\frac{\sigma \lambda}{R-\lambda} \hat{\lambda}-\frac{R \lambda}{R-\lambda} \hat{\lambda}\right)=\frac{\sigma \lambda}{R-\lambda}(\sigma-R) \hat{\lambda}<0,
$$

where the inequality follows because $\sigma<1<R$. Thus, we see a boom-bust in capital and thus in output. 
Plugging into the capital equation above we have

$$
\begin{aligned}
\frac{R \eta}{r} \hat{q}_{0} & =\left(\frac{1}{1-\underline{\beta \sigma}}\right) \hat{\lambda}\left(\frac{\sigma \lambda}{R-\lambda}\right)-\hat{\lambda}\left(\frac{\underline{\beta} \sigma}{1-\underline{\beta \sigma} \sigma}\right)\left(\frac{\lambda}{1-\underline{\beta} \lambda}\right), \\
& =\hat{\lambda}\left(\frac{1}{1-\underline{\beta \sigma}}\right)\left(\frac{\sigma \lambda}{R-\lambda}-\underline{\beta} \sigma\left(\frac{R \lambda}{R-\lambda}\right)\right), \\
& =\lambda \hat{\lambda}\left(\frac{1}{1-\underline{\beta} \sigma}\right)\left(\frac{\sigma}{R-\lambda}-\frac{\sigma}{R-\lambda}\right)=0 \\
& \Longrightarrow \hat{q}_{0}=0 .
\end{aligned}
$$

Financial Shocks and News Now suppose that the financial shock occurs in period $t=N$ and agents learn of the shock at $t=0$. First, linearizing the budget constraints with news implies.

$$
\hat{K}_{s}= \begin{cases}\sigma^{s} \hat{K}_{0} & 0<s<N \\ \sigma^{s} \hat{K}_{0}+\frac{\sigma \delta \hat{z}}{R-\delta} & s=N \\ \sigma^{s} \hat{K}_{0}+\sigma^{s-N} \frac{\delta \hat{z}}{R-\delta}(\sigma-R) & s>N\end{cases}
$$

Therefore we are able to calculate $\hat{q}_{0}$

$$
\begin{aligned}
& \hat{q}_{0}=\frac{r}{R \eta} \sum_{s=0}^{\infty} R^{-s} \hat{K}_{s}, \\
& \hat{q}_{0}=\frac{r}{R \eta}\left(\sum_{s=0}^{\infty} R^{-s} \sigma^{s} \hat{K}_{0}+\sum_{s=N}^{\infty} R^{-s} \sigma^{s-N+1} \frac{\delta \hat{z}}{R-\delta}-\sum_{s=N+1}^{\infty} R^{-s} \sigma^{s-N} \frac{\delta R \hat{z}}{R-\delta}\right), \\
& \hat{q}_{0}=\frac{r}{R \eta} \sum_{s=0}^{\infty} R^{-s} \sigma^{s} \hat{K}_{0} .
\end{aligned}
$$

Plugging in the budget constraint (37) at $t=0$ we have

$$
\begin{aligned}
& \hat{K}_{0}\left(\frac{\delta}{\eta}+\frac{R-\delta}{r}\right) \frac{r}{\delta R}=\frac{r}{R \eta} \sum_{s=0}^{\infty} R^{-s} \sigma^{s} \hat{K}_{0} \\
& \hat{K}_{0}\left(1+\frac{\eta(R-\delta)}{\delta r}\right)=\frac{\hat{K}_{0}}{1-\frac{\sigma}{R}} \Longrightarrow \hat{K}_{0}=0 .
\end{aligned}
$$

For a shock occurring at time $t=N$, then for $s<N, \hat{K}_{s}=0$, and

$$
\hat{K}_{N}=\frac{\sigma \delta \hat{z}}{R-\delta}>0, \quad \hat{K}_{N+1}=\frac{\sigma \delta \hat{z}}{R-\delta}(\sigma-R)<0, \quad \hat{K}_{N+s}=\frac{\sigma^{s} \delta \hat{z}}{R-\delta}(\sigma-R)<0 .
$$


For $s \leq N, \hat{q}_{s}=0$, and for $s>N, \hat{q}_{s}<0$.

\section{Behavioral Shock Proof}

Proof of Proposition 4. Note that the linearized budget constraint at $t=1$ becomes

$$
\hat{\hat{u}}_{1}+\hat{\mathrm{K}}_{1}=\hat{K}_{0}+\frac{R}{r}\left(\hat{\hat{q}}_{1}-\hat{q}_{1}\right),
$$

reflecting that capital and debt were predetermined. It's useful to re-write this as

$$
\left(1+\frac{1}{\eta}\right) \hat{K}_{1}=\frac{R}{r} \hat{q}_{1}+Z
$$

where $Z \equiv \hat{K}_{0}-\frac{R}{r} \hat{q}_{1}=\underline{\beta} \gamma \hat{z}-\frac{R}{r} r \underline{\beta} \gamma \hat{z}=-\gamma(1-\underline{\beta}) \hat{z}$. It is as if experts face a negative productivity shock. They have more capital than steady state, $\hat{K}_{0}>0$, but also more debt, $\hat{q}_{1}>0$, and the additional debt weighs on available funds by more than the additional output from higher capital.

We can write the capital price, which is the discounted value of future user costs, as

$$
\hat{q}_{1}=\frac{r}{R \eta} \frac{\hat{\hat{K}}_{1}}{1-\underline{\beta} \gamma} .
$$

Plugging into the budget constraint, we therefore have

$$
\begin{aligned}
\left(1+\frac{1}{\eta}\right) \hat{\hat{K}}_{1} & =\frac{1}{\eta} \frac{\hat{\hat{K}}_{1}}{1-\underline{\beta} \gamma}+Z, \\
(1+\eta)(1-\underline{\beta} \gamma) \hat{\hat{K}}_{1} & =\hat{\hat{K}}_{1}+\eta(1-\underline{\beta} \gamma) Z, \\
\hat{K}_{1} & =\frac{\eta(1-\underline{\beta} \gamma)}{(1+\eta)(1-\underline{\beta} \gamma)-1} Z, \\
\hat{\mathrm{K}}_{1} & =\frac{\eta(1-\underline{\beta} \gamma)}{1+\eta-\underline{\beta} \eta-1} Z, \\
\hat{\mathrm{K}}_{1} & =\frac{\eta(1-\underline{\beta} \gamma)}{\eta(1-\underline{\beta})} Z, \\
\hat{\mathrm{K}}_{1} & =-\frac{(1-\underline{\beta} \gamma)}{(1-\underline{\beta})} \gamma(1-\underline{\beta}) \hat{z}, \\
\hat{\mathrm{K}}_{1} & =-(1-\underline{\beta} \gamma) \gamma \hat{z}=\hat{K}_{1} .
\end{aligned}
$$


Hence, the experts' capital holdings in the new equilibrium is exactly as it would have been. The asset price, however, is lower

$$
\hat{\hat{q}}_{1}=-\frac{r}{R \eta} \frac{(1-\underline{\beta} \gamma) \gamma \hat{z}}{1-\underline{\beta} \gamma}=-\frac{r \underline{\beta}}{1+\eta} \hat{z}<0 .
$$

Recall that $\hat{q}_{1}>0$.

News about expert productivity Finally, suppose that at $t=0$ agents learn that experts will have additional productivity $\Delta$ at $t=1$. We can write the asset price at $t=1$ as a function of capital at $t=1$ as

$$
\hat{q}_{1}=\frac{r}{R \eta}\left(\frac{1}{1-\beta \gamma}\right) \hat{K}_{1}
$$

Plugging in for the value of capital at $t=1$ we have

$$
\hat{q}_{1}=\frac{r}{R \eta}\left(\frac{1}{1-\beta \gamma}\right)\left(\gamma \Delta+\gamma \hat{K}_{0}\right)=\frac{r}{R \eta}\left(\frac{\gamma}{1-\beta \gamma}\right)\left(\Delta+\hat{K}_{0}\right) .
$$

From the equation for the asset price at $t=0$, we have

$$
\begin{aligned}
\hat{q}_{0} & =\frac{r}{R \eta} \hat{K}_{0}+\frac{r}{R \eta}\left(\frac{\beta \gamma}{1-\beta \gamma}\right)\left(\Delta+\hat{K}_{0}\right) \\
& =\frac{r}{R \eta}\left(\frac{1}{1-\beta \gamma}\right) \hat{K}_{0}+\frac{r}{R \eta}\left(\frac{\beta \gamma}{1-\beta \gamma}\right) \Delta .
\end{aligned}
$$

Plugging in the budget constraint at $t=0$, we have

$$
\hat{K}_{0}=\left(\frac{1}{r(1+\eta)}\right) \Delta
$$

Plugging this value into the budget constraint equation to get the asset price at $t=0$, we have

$$
\hat{q}_{0}=\left(\frac{\beta}{\eta}\right) \Delta
$$

and then we have

$$
\hat{q}_{1}=\frac{R}{1+\frac{1}{\eta}}\left(\frac{\beta}{\eta}\right) \Delta=\frac{\Delta}{1+\eta} .
$$


Finally, we have

$$
\hat{K}_{1}=\gamma \Delta+\gamma\left(\frac{1}{r(1+\eta)}\right) \Delta=\gamma\left(1+\frac{1}{r(1+\eta)}\right) \Delta .
$$

Plugging in $\hat{q}_{1}$ and $\hat{K}_{0}$ into equation (43) it is clear that we end up with $\hat{K}_{1}<0<\hat{K}_{1}$ implying different capital dynamics. 